\title{
MYC-dependent oxidative metabolism regulates osteoclastogenesis via nuclear receptor ERRa
}

\author{
Seyeon Bae, ${ }^{1}$ Min Joon Lee, ${ }^{1}$ Se Hwan Mun, ${ }^{1}$ Eugenia G. Giannopoulou, ${ }^{1,2}$ Vladimir Yong-Gonzalez, ${ }^{3}$ Justin R. Cross, ${ }^{3}$ \\ Koichi Murata, ${ }^{1}$ Vincent Giguère, ${ }^{4}$ Marjolein van der Meulen, ${ }^{5,6}$ and Kyung-Hyun Park-Min ${ }^{1,7}$

\begin{abstract}
'Arthritis and Tissue Degeneration Program, David Z. Rosensweig Genomics Research Center, Hospital for Special Surgery, New York, New York, USA. Biological Sciences Department, New York City College of Technology, City University of New York, Brooklyn, New York, USA. ${ }^{3}$ Donald B. and Catherine C. Marron Cancer Metabolism Center, Memorial Sloan Kettering Cancer Center, New York, New York, USA. ${ }^{4}$ Coodman Cancer Research Centre, McGill University, Montréal, Canada; Departments of Biochemistry, Medicine and Oncology, McGill University, Montréal, Canada. ${ }^{5}$ Sibley School of Mechanical and Aerospace Engineering, Cornell University, Ithaca, New York, USA. ${ }^{6}$ Musculoskeletal Integrity Program, Hospital for Special Surgery, New York, New York, USA. `Department of Medicine, Weill Cornell Medical
\end{abstract} \\ College, New York, New York, USA.
}

\begin{abstract}
Osteoporosis is a metabolic bone disorder associated with compromised bone strength and an increased risk of fracture. Inhibition of the differentiation of bone-resorbing osteoclasts is an effective strategy for the treatment of osteoporosis. Prior work by our laboratory and others has shown that MYC promotes osteoclastogenesis in vitro, but the underlying mechanisms are not well understood. In addition, the in vivo importance of osteoclast-expressed MYC in physiological and pathological bone loss is not known. Here, we have demonstrated that deletion of Myc in osteoclasts increases bone mass and protects mice from ovariectomy-induced (OVX-induced) osteoporosis. Transcriptomic analysis revealed that MYC drives metabolic reprogramming during osteoclast differentiation and functions as a metabolic switch to an oxidative state. We identified a role for MYC action in the transcriptional induction of estrogen receptor-related receptor $\alpha$ (ERR $\alpha$ ), a nuclear receptor that cooperates with the transcription factor nuclear factor of activated T cells, c1 (NFATc1) to drive osteoclastogenesis. Accordingly, pharmacological inhibition of ERR $\alpha$ attenuated OVX-induced bone loss in mice. Our findings highlight a MYC/ ERR $\alpha$ pathway that contributes to physiological and pathological bone loss by integrating the MYC/ERR $\alpha$ axis to drive metabolic reprogramming during osteoclast differentiation.
\end{abstract}

\section{Introduction}

MYC (also known as c-MYC) is a broadly acting transcription factor that regulates the differentiation and proliferation program of cells by multiple mechanisms including a transcriptional amplification of target genes (1-3). MYC deficiency in mice is embryonically lethal (4), and aberrant overexpression of MYC is found in human cancers (5). MYC has been implicated in in vitro osteoclastogenesis $(6,7)$, and we recently identified MYC as an important transcriptional factor of the RANKL-induced transcriptional program by directly regulating nuclear factor of activated T cells, c1 (NFATc1) in osteoclasts (8). However, the molecular and cellular mechanism by which MYC regulates osteoclastogenesis and the functional role of myeloid cell-specific MYC during physiological and pathological bone remodeling in vivo are not completely elucidated.

Osteoclasts are multinuclear giant cells responsible for bone resorption and are derived from the myeloid lineage cells in response to RANKL $(9,10)$. RANKL activates canonical signaling pathways including NF- $\mathrm{KB}$ and MAPK as well as immunoreceptor tyrosine-

\section{Related Commentary: p. 2530}

Authorship note: S. Bae and M.J. Lee contributed equally to this work. Conflict of interest: The authors have declared that no conflict of interest exists. Submitted: August 3, 2016; Accepted: March 23, 2017. Reference information: J Clin Invest. 2017;127(7):2555-2568. https://doi.org/10.1172/JCl89935 based activation motif (ITAM) immunoreceptor signaling pathways, synergistically inducing the expression of NFATc1, a master regulator of osteoclastogenesis (11). Although NFATc1 is known as an essential factor regulating osteoclast-specific gene expression programs (11, 12), NFATc1 deficiency affects only a subset of RANKL-regulated transcriptional programs (13), implicating the importance of other transcription factors in gene regulation during osteoclast differentiation. Recently, association of the comprehensive transcriptional factor network with molecular pathways in osteoclasts has gained much interest, and identification of new transcriptional pathways controlling osteoclastogenesis will offer promising therapeutic targets for the treatment of pathological bone disorders such as osteoporosis.

The importance of metabolic reprogramming in osteoclast differentiation is increasingly appreciated. Metabolic adaptation to meet increased energy demand is required to undergo osteoclast differentiation $(7,14-17)$. Recent studies have shown that several factors such as peroxisome proliferator-activated receptor $\gamma$ coactivator $1 \beta$ (PGC1 $\beta$ ), PPAR $\gamma$, and estrogen receptor-related receptor $\alpha(\mathrm{ERR} \alpha)$ govern key metabolic processes by transcriptionally regulating distinct metabolic genes during osteoclast differentiation as part of bone remodeling (18-21). However, whether there exists a key upstream regulator that controls these factors during RANKL-induced metabolic reprogramming is not clear.

In this study, we demonstrate that MYC and MYC-mediated regulation of oxidative respiration provide potential links between RANK signaling and metabolic reprogramming during 
A

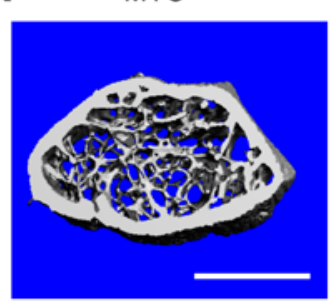

$M^{M} C^{M M}$

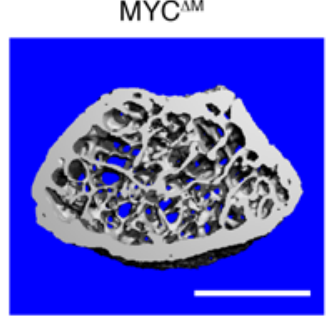

C

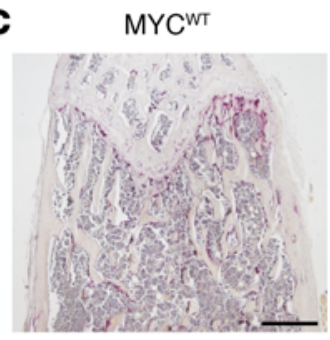

$M^{M} C^{M M}$

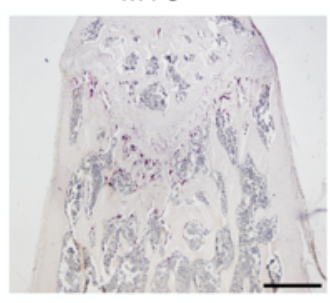

B
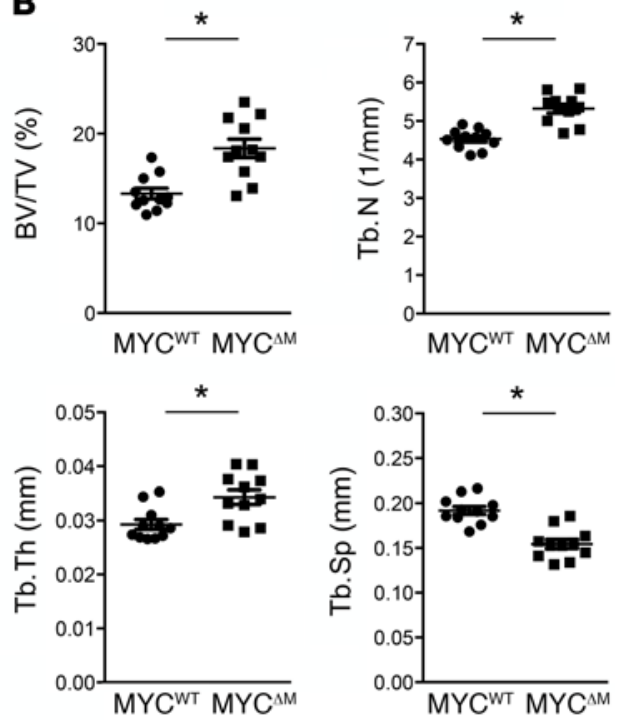

D
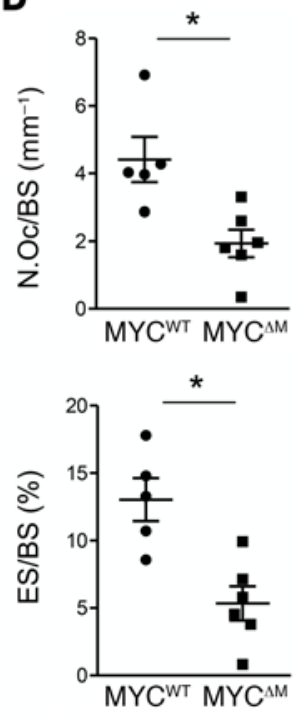

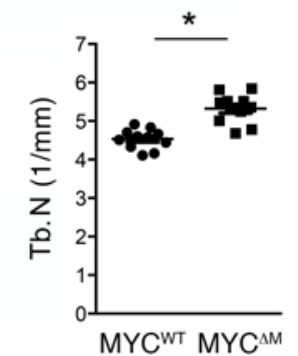

Figure 1. Myeloid-specific MYC-deficient mice have high bone mass. (A and $\mathbf{B}$ ) $\mu \mathrm{CT}$ analysis of femurs from 12-week-old male MYC ${ }^{\triangle M}$ and littermate control MYCWT mice ( $n=11$ per group). (A) Representative images show trabecular architecture by $\mu \mathrm{CT}$ reconstruction in the distal femurs. Scale bars: $1 \mathrm{~mm}$. (B) $\mu \mathrm{CT}$ measurements for the indicated parameters in distal femurs. Bone volume/tissue volume ratio (BV/ TV), trabecular numbers (Tb.N), trabecular thickness (Tb.Th ), and trabecular space (Tb.Sp) were determined by $\mu \mathrm{CT}$ analysis. (C and $\mathbf{D})$ Histomorphometric analysis of femurs from 12-week-old male $\mathrm{MYC}^{\Delta \mathrm{M}}$ and MYCWT mice $(n \geq 5)$. (C) Representative images showing TRAP-positive, multinucleated osteoclasts (red). Scale bars: $200 \mu \mathrm{m}$. (D) Plots show the number of osteoclasts per bone surface (N.Oc/BS), osteoclast surface area per bone surface (Oc.S/BS), eroded surface per bone surface (ES/BS), and osteoblast surface area per bone surface (Ob.S/BS). All data are shown as the mean \pm SEM. ${ }^{*} P<0.05$, by 2 -tailed, unpaired $t$ test in $\mathbf{B}$ and $\mathbf{D}$. osteoclastogenesis and identify a MYC/ERR $\alpha$ pathway that drives metabolic reprogramming during osteoclast differentiation as an important regulator of physiological and pathological bone loss. Moreover, our study highlights the possibility that pharmacological inhibition of ERR $\alpha$ may offer translational potential for the treatment of human bone disorders such as osteoporosis.

\section{Results}

Myeloid-specific deletion of MYC causes increased bone mass and decreased osteoclastogenesis. MYC was transiently expressed in the early stage of osteoclastogenesis (within 24 hours after RANKL stimulation) in both human and mouse osteoclast precursors (OCPs) (Supplemental Figure 1; supplemental material available online with this article; https://doi.org/10.1172/JCI89935DS1). To determine the role of this transient MYC expression in OCPs in physiological bone remodeling, we first investigated the bone phenotype of $M y c$ conditional knockout mice generated by crossing $M y c^{f l / f l}$ mice with LysM-Cre mice (22) to specifically delete $M y c$ in myeloid/osteoclast lineage cells (hereafter referred to as $\mathrm{MYC}^{\Delta \mathrm{M}}$ mice). Microcomputed tomography ( $\mu \mathrm{CT})$ analysis of 12-week-old male and female $\mathrm{MYC}^{\Delta \mathrm{M}}$ mice clearly indicated increased bone mass compared with that seen in their littermate controls (LysMCre, hereafter referred to as MYC ${ }^{\mathrm{WT}}$ mice) (Figure 1, A and B, and Supplemental Figure 2, A and B). The body weight was not affected by $M y c$ deletion in either male or female mice (Supplemental Figure 2C). Bone histomorphometric analysis revealed significantly decreased trabecular osteoclast numbers and surface area as well as bone resorption activity (eroded surface area) in $\mathrm{MYC}^{\Delta \mathrm{M}}$ mice, whereas the osteoblast surface areas were comparable (Figure $1, \mathrm{C}$ and $\mathrm{D}$ ). These results suggest that the high-bone-mass phenotype of $\mathrm{MYC}^{\Delta \mathrm{M}}$ mice was caused by compromised osteoclast activity and that MYC plays an important role in physiological bone remodeling.

Next, we isolated OCPs from $\mathrm{MYC}^{\Delta \mathrm{M}}$ bone marrow and evaluated in vitro osteoclast differentiation in the presence of macrophage-CSF (M-CSF) and RANKL. MYC was efficiently diminished in MYC-deficient OCPs (Supplemental Figure 3). MYC deficiency resulted in essentially a complete failure of OCPs to differentiate 
A

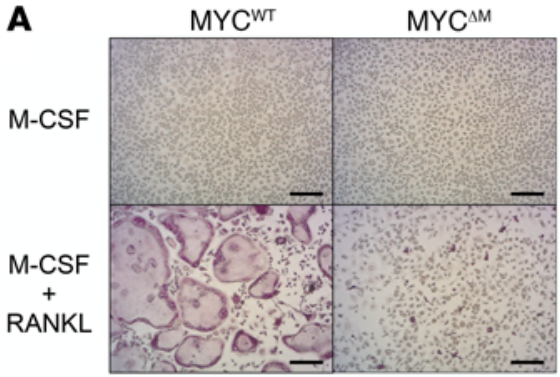

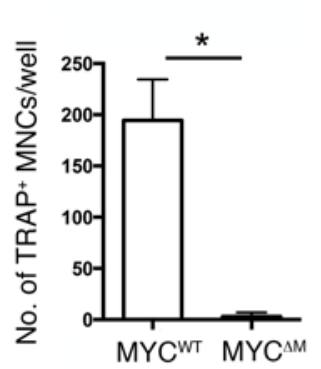

B

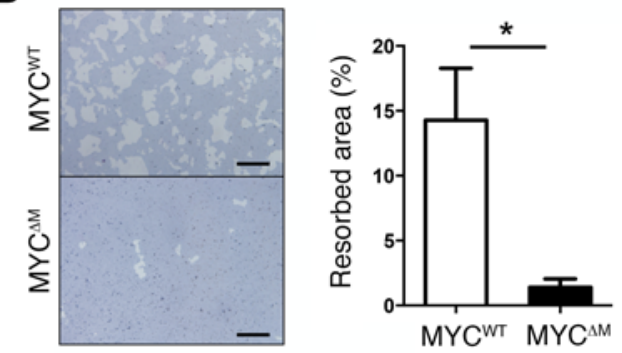

C<smiles></smiles>

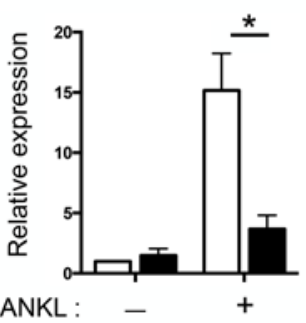

$\operatorname{ltg} 33$

Ctsk
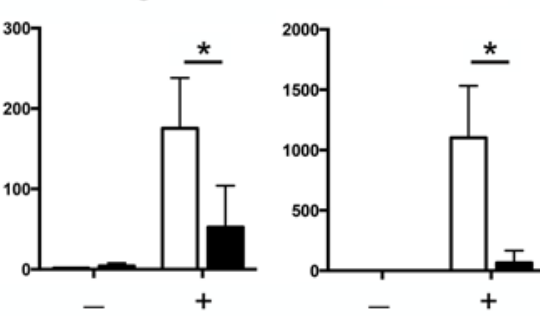

\section{Dcstamp}

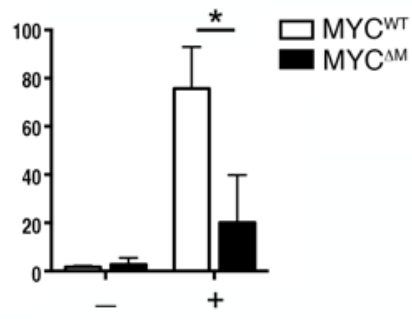

D

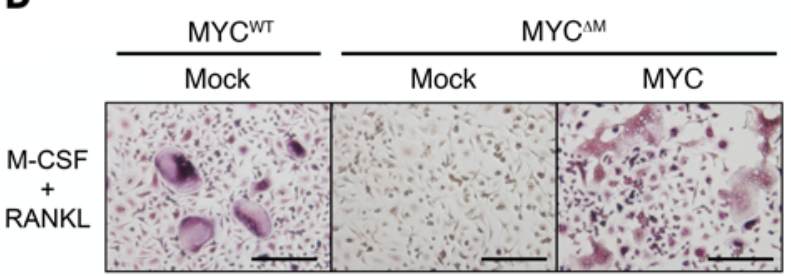

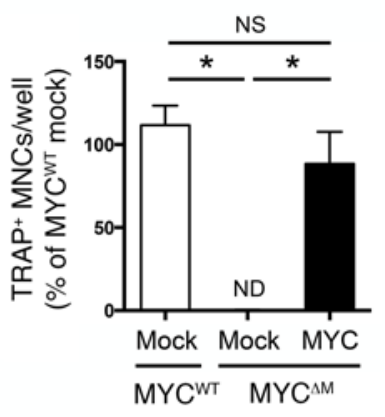

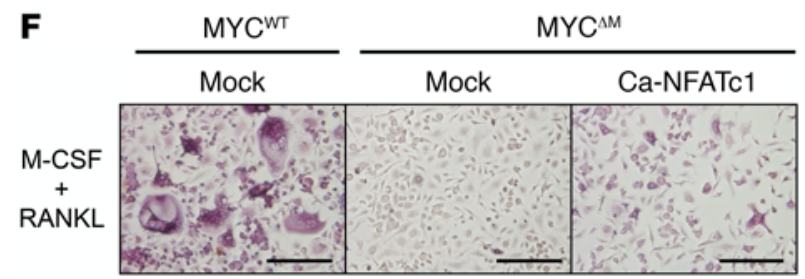

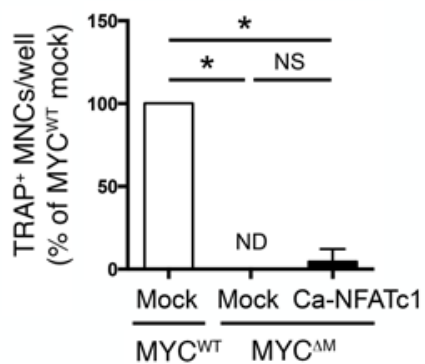

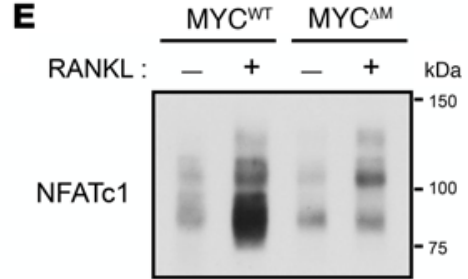

$\alpha$-Tubulin

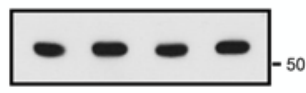

Figure 2. MYC is important for osteoclast differentiation and resorption. (A) Representative images show osteoclast differentiation in control (MYCWT) and MYC-deficient (MYC ${ }^{\triangle M}$ ) OCPs stimulated with RANKL (50 ng/ml). Scale bars: $200 \mu \mathrm{m}$. Graph depicts the number of TRAP-positive multinucleated cells (MNCs) counted in triplicate from 5 independent experiments. (B) Representative images show bone resorption activity of MYCWT and MYC ${ }^{\triangle M}$ OCPs stimulated with RANKL. Scale bars: $200 \mu \mathrm{m}$. Graph depicts the percentage of resorbed pit area per total area from at least 3 experiments. (C) Expression of osteoclast-related genes (relative to the Hprt housekeeping gene) on day 3 after RANKL stimulation $(n=8)$. (D) Osteoclast differentiation in mock-infected or MYC-transduced MYC-deficient OCPs stimulated with RANKL $(50 \mathrm{ng} / \mathrm{ml})$. Representative images of TRAP-stained cells are shown. Scale bars: $200 \mu \mathrm{m}$. Graph shows the number of TRAP-positive MNCs counted in triplicate from at least 4 experiments. (E) Immunoblot of whole-cell lysates using NFATc 1 and $\alpha$-tubulin antibodies. $\alpha$-Tubulin served as a loading control. Data are representative of 3 experiments. (F) Osteoclast differentiation in mock-infected or Ca-NFATc1-transduced MYC-deficient OCPs. Representative images of TRAP-stained cells are shown. Scale bars: $200 \mu \mathrm{m}$. Graph shows the number of TRAP-positive MNCs counted in triplicate from at least 3 experiments. All data are shown as the mean \pm SEM. ${ }^{*} P<0.05$, by 2 -tailed, unpaired $t$ test (A and B); 2 -way ANOVA with Tukey's post-hoc test (C); 1-way ANOVA with Tukey's post-hoc test (D and F). ND, not detected.

into multinucleated tartrate-resistant acid phosphatase-positive (TRAP-positive) cells that resorb bone (Figure 2, A and B). Accordingly, RANKL-induced expression of osteoclast marker genes such as Itgb3 (encodes $\beta 3$ integrin), Ctsk (encodes cathepsin K), and Dcstamp (encodes dendrocyte-expressed seven-transmembrane protein) was strongly suppressed in MYC-deficient OCPs (Figure
2C). Ectopic expression of MYC rescued the defect in osteoclastogenesis in MYC-deficient OCPs (Figure 2D), thus confirming that deletion of $M y c$ is responsible for the loss of osteoclastogenesis. We initially reasoned that the complete loss of osteoclastogenesis could be explained by decreased bone marrow osteoclast progenitor cells in $\mathrm{MYC}^{\Delta \mathrm{M}}$ mice or their decreased proliferation in vitro. 
A

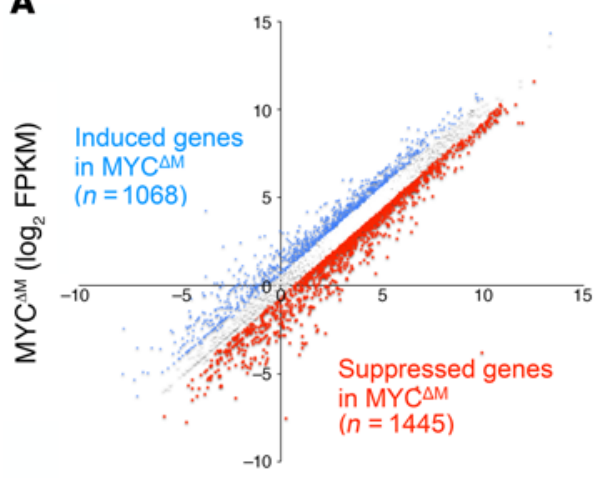

MYC ${ }^{W T}\left(\log _{2}\right.$ FPKM)
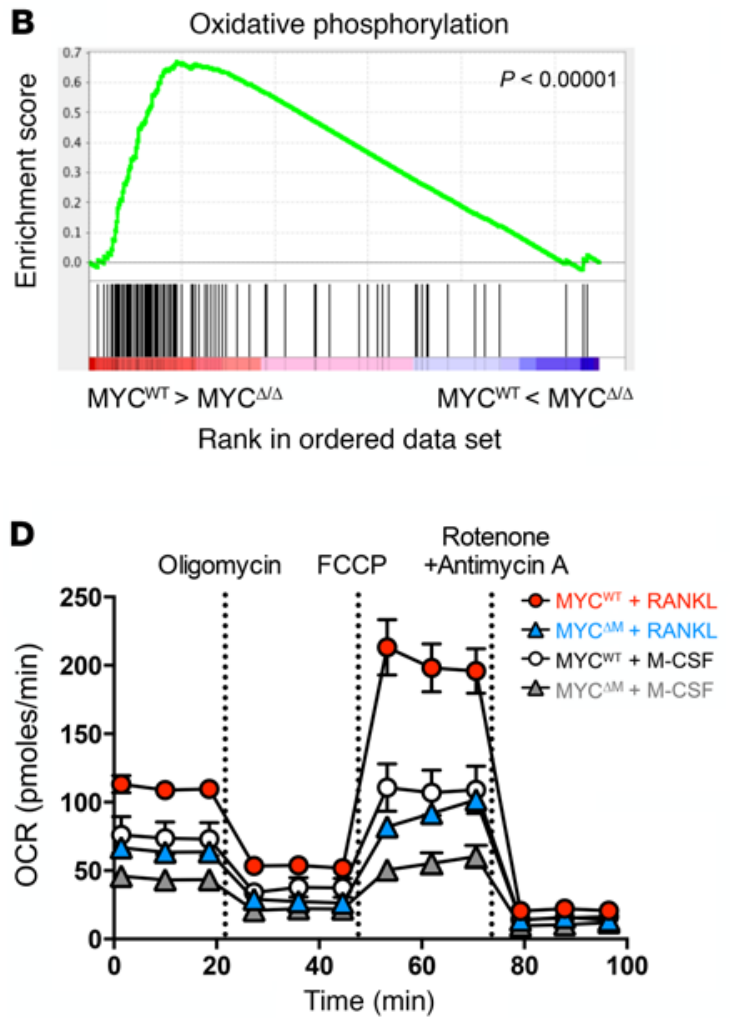

E Basal respiration

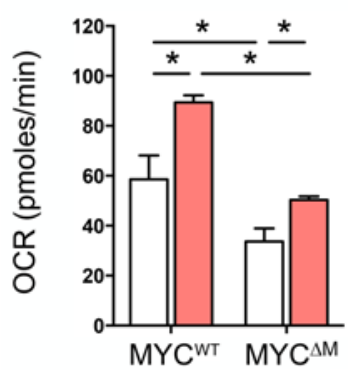

C
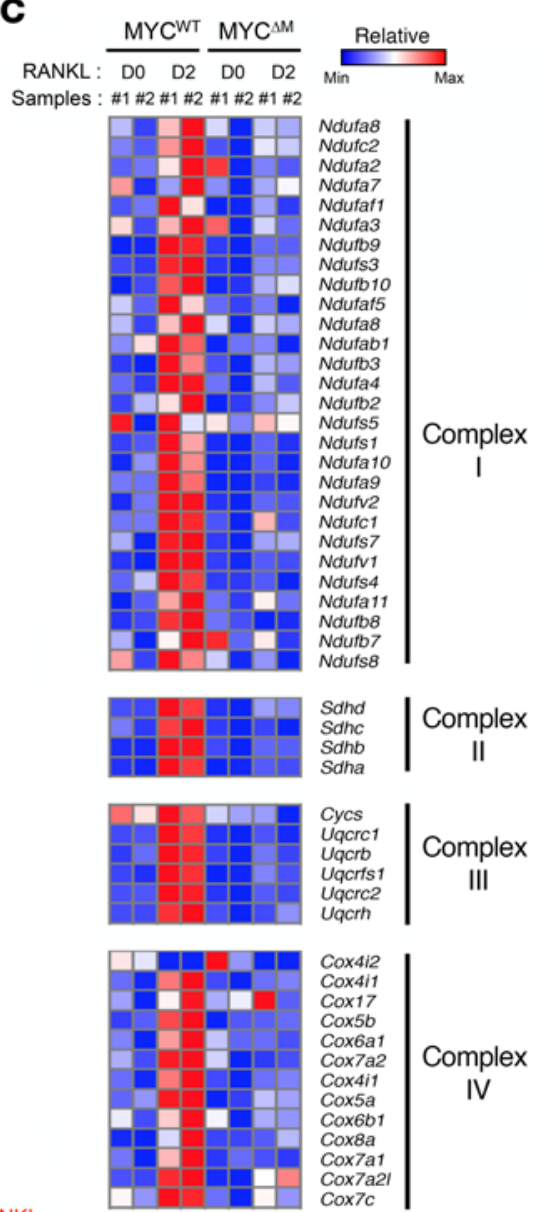
Uacre2 Uqcrh

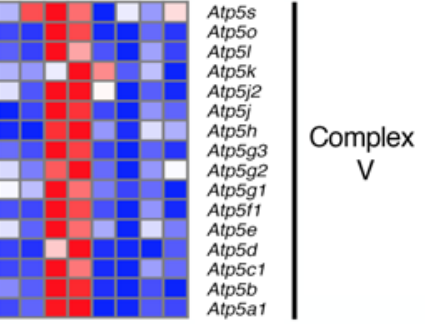

$\mathbf{F}$
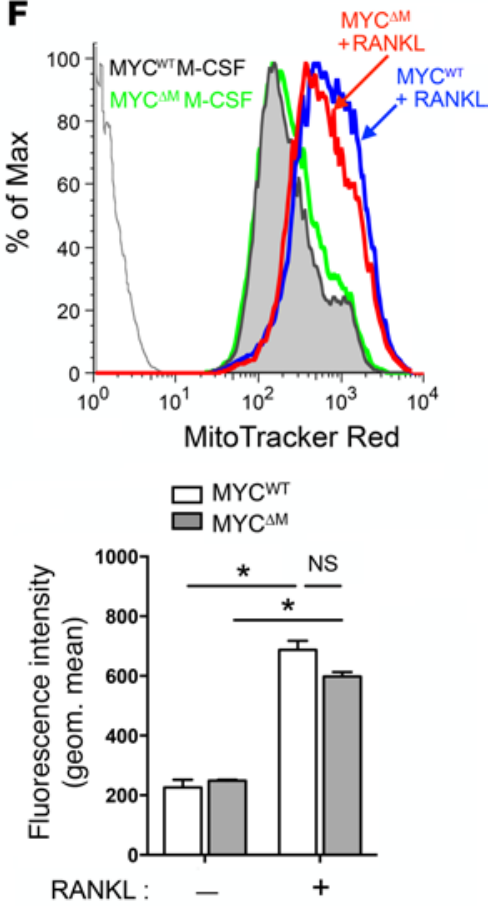

G Basal respiration

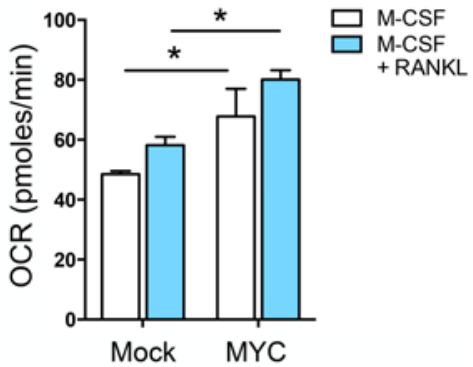

Respiratory capacity

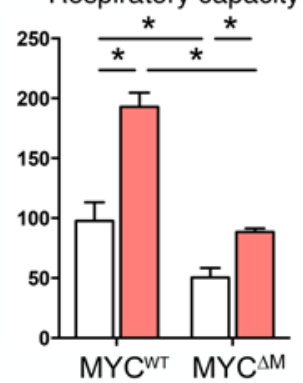

Respiratory reserve

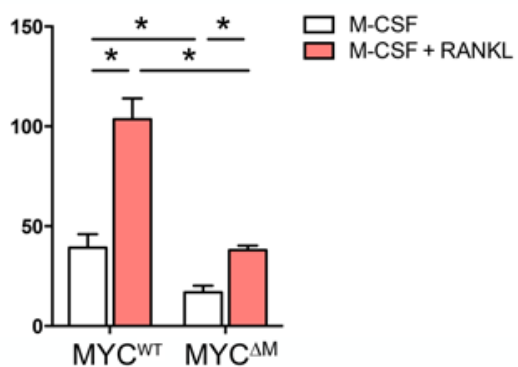


Figure 3. MYC deficiency impairs mitochondrial respiration during osteoclast differentiation. Control $\left(M Y C^{\mathrm{WT}}\right)$ and MYC-deficient $\left(M Y C^{\triangle M}\right)$ OCPs were stimulated with RANKL $(50 \mathrm{ng} / \mathrm{ml})$ for 2 days. (A) Scatterplot of global gene expression profiles of $M Y C^{W T}$ and $M Y C^{\triangle M}$ OCPs derived from RNA-seq analysis. (B) GSEA of RANKL-stimulated MYC ${ }^{\triangle M}$ OCPs, with genes ranked on the basis of expression in MYC ${ }^{\triangle \mathrm{M}}$ OCPs relative to that in MYCWT OCPs, showing the distribution of genes in the oxidative phosphorylation gene set against the ranked list of the genes from the RNA-seq analysis. (c) Heatmap of RNA-seq FPKM values for genes involved in oxidative phosphorylation in MYCWT and MYC ${ }^{\triangle M}$ OCPs following RANKL stimulation for 2 days. RNA-seq data from 2 biological replicates were used. DO, day 0 without RANKL stimulation; D2, day 2 following RANKL stimulation; Min, minimum; Max, maximum. (D and $\mathbf{E}$ ) Mitochondrial function was assessed by real-time OCR measurement after sequential treatment of compounds modulating mitochondrial function. The OCR was normalized to the relative amount of DNA. (D) Representative time course data. (E) Assessment of mitochondrial activity as described in Supplemental Figure $9(n=3)$. (F) Measurements of mitochondrial mass using MitoTracker Red with flow cytometry $(n=3)$. (G) The basal OCR was measured with mock-infected or MYC-transduced MYC-deficient OCPs stimulated with RANKL (50 ng/ $\mathrm{ml}$ ) for 2 days $(n=3)$. All data are shown as the mean \pm SEM. ${ }^{*} P<0.05$, by 2-way ANOVA with Tukey's post-hoc test.

However, consistent with a previous report showing intact bone marrow and lymphoid compartments in $M y c$-knockout mice (23), the total number and frequency of the $\mathrm{CD} 11 \mathrm{~b}{ }^{-/ 1} \mathrm{Ly} 6 \mathrm{C}^{\text {hi }}$ osteoclast progenitor population (24) residing in the bone marrow were comparable between $\mathrm{MYC}^{\Delta \mathrm{M}}$ and MYC ${ }^{\mathrm{WT}}$ mice (Supplemental Figure 4, A and B). In addition, MYC deficiency had modest effects on apoptosis, and MYC-deficient OCPs showed decreased proliferation. These results could not explain the striking effects of MYC deficiency on osteoclastogenesis (Supplemental Figure 4, C and D). Furthermore, RANK expression and proximal RANKL signaling were not altered in MYC-deficient OCPs (Supplemental Figure 5). Taken together, these results indicate that RANKL-inducible MYC is crucial for osteoclast differentiation in vitro.

MYC is a crucial regulator of oxidative metabolism during osteoclastogenesis. Consistent with our previous results showing MYC as a direct regulator of NFATc1 (8), expression levels of both NFATc1 mRNA and protein were suppressed in MYC-deficient OCPs (Figure 2, C and E). We first reasoned that restoration of NFATc1 activity would compensate for impaired osteoclastogenesis in MYC-deficient OCPs. To test this notion, we transduced MYC-deficient OCPs with a retrovirus encoding the constitutively active form of NFATc1 (Ca-NFATc1). With RANKL stimulation, the expression of NFATc1 and $\beta 3$ integrin, a downstream target of NFATc1 (12), in Ca-NFATc1transduced MYC-deficient OCPs was comparable to that in control OCPs (Supplemental Figure 6), but was not sufficient to rescue the defective osteoclastogenesis seen in MYC-deficient OCPs (Figure $2 \mathrm{~F}$ ), suggesting that MYC regulates alternative pathways that are required to cooperate with NFATc1 to promote osteoclastogenesis.

To find new pathways and mechanisms regulated by MYC during osteoclastogenesis, we performed an unbiased transcriptomic analysis using high-throughput RNA sequencing (RNA-seq) with 2 biological replicates after stimulating cells with RANKL for 2 days (Supplemental Figure 7). A total of 1,068 genes were induced ( $>1.5$ fold) and 1,445 genes were suppressed ( $>1.5$ fold) in MYC-deficient OCPs compared with control OCPs (Figure 3A). We used gene set enrichment analysis (GSEA) (25) to broadly test for enrichment of well-defined gene sets from the comprehensive
Molecular Signature Data Base (MSigDB), version 5.1 (www.broadinstitute.org) and Ingenuity Pathway Analysis (IPA) for MYC-dependent genes, which are downregulated in $\mathrm{MYC}^{\triangle \mathrm{M}} \mathrm{OCPs}$ relative to MYC ${ }^{\text {WT }}$ OCPs on day 2 after RANKL stimulation. Genes associated with metabolic pathways, including oxidative phosphorylation and the TCA cycle, were significantly enriched in MYC-dependent genes (Table 1, Figure 3B, and Supplemental Figure 8). Accordingly, metabolic genes involved in oxidative phosphorylation, which are highly induced in MYC ${ }^{\mathrm{WT}}$ OCPs upon RANKL stimulation, were suppressed in $\mathrm{MYC}^{\Delta \mathrm{M}}$ OCPs (Figure 3C).

Recent studies show that a metabolic shift toward oxidative processes during osteoclast differentiation provides metabolites (15-17), although key upstream modulators that induce oxidative metabolic reprogramming in osteoclastogenesis are not yet clarified. To directly test whether MYC mediates the RANKL-induced shift toward mitochondrial respiration, we performed an extracellular flux analysis 2 days after RANKL stimulation (Figure 3, D and $\mathrm{E}$, and Supplemental Figure 9). RANKL stimulation led to a characteristic increase in the basal oxygen consumption rate (OCR) in control OCPs (Figure 3, D and E). Strikingly, the basal OCR and other mitochondrial activities such as ATP production, respiratory capacity (maximal electron transport chain activity), and respiratory reserve (flexibility with increased energy demand) were severely impaired in MYC-deficient OCPs upon RANKL stimulation (Figure 3, D and E). We confirmed that the RANKL-induced increase in mitochondrial mass was comparable between MYC-deficient and control OCPs (Figure 3F), indicating that the impaired mitochondrial respiration in MYC-deficient OCPs was not caused by a defect in the quantity of mitochondria. Moreover, the basal OCR was greatly increased by the ectopic expression of MYC (Figure $3 G)$. Consistent with previous reports $(7,16)$, perturbation of metabolic pathways such as glutaminolysis, glycolysis, and oxidative phosphorylation using specific inhibitors severely abrogated osteoclast differentiation (Supplemental Figure 10), implicating the significance of metabolic reprogramming in osteoclastogenesis. Collectively, these results indicate that MYC is a crucial regulator of RANKL-activated mitochondrial respiration that supports an increased energy demand during osteoclast differentiation.

$M Y C$ regulates ERR $\alpha$ during osteoclast differentiation. MYC expression is kept at low levels in normal cells via accelerated decay mechanisms, and aberrant expression of MYC causes tumorigenesis (5). In osteoclastogenesis, MYC expression is transient (Supplemental Figure 1), and thus we hypothesized that MYC induces downstream transcription factors to directly activate osteoclast-related genes and sustain osteoclast differentiation. To discover these factors, we used GSEA to identify transcription factor-binding motifs that are enriched in the promoters of MYC-dependent genes, which can be regulated directly by MYC, or indirectly via MYC-induced transcription factors. In addition to recovering the MYC-binding motif, which validated the approach, and the NFAT-binding motif, as expected on the basis of our previous work (8), we found highly significant enrichment of binding motifs for Sp1 transcription factor (SP1), estrogen-related receptor (ERR), MYC-associated zinc finger protein (MAZ), and splicing factor 1 (SF1) (Table 2); of these, only estrogen-related receptor $\alpha$ (Esrra, encoding ERR $\alpha$ ) was dynamically regulated during osteoclast differentiation (Figure 4A). 


\section{Table 1. Gene ontology analysis of MYC ${ }^{W T}$ and MYC ${ }^{\triangle M}$ OCPs derived from RNA-seq analysis}

\begin{tabular}{ll} 
Gene set & $P$ value \\
Suppressed genes in MYC ${ }^{\mathrm{MM}}$ OCPs & \\
\hline KEGG_Ribosome & $9.73 \times 10^{-91}$ \\
\hline KEG__Oxidative phosphorylation & $1.54 \times 10^{-45}$ \\
\hline KEGG_Parkinsons disease & $3.21 \times 10^{-39}$ \\
\hline KEGG_Alzheimers disease & $2.28 \times 10^{-30}$ \\
\hline KEGG_Citrate cycle TCA cycle & $4.52 \times 10^{-10}$ \\
\hline Induced genes in MYC ${ }^{\Delta M}$ OCPs & \\
\hline KEGG_MAPK signaling pathway & $2.75 \times 10^{-16}$ \\
\hline KEGG_Pathways in cancer & $2.94 \times 10^{-12}$ \\
\hline KEGG_Chemokine signaling & $2.45 \times 10^{-11}$ \\
\hline KEGG_B cell receptor signaling & $1.72 \times 10^{-10}$ \\
\hline KEG__Focal adhesion & $2.74 \times 10^{-10}$ \\
\hline
\end{tabular}

KEGG, Kyoto Encyclopedia of Genes and Genomes.

$\mathrm{ERR} \alpha$ is not an estrogen receptor, but instead is a nuclear receptor implicated in increasing cellular energy metabolism in tissues such as liver and skeletal muscle in response to environmental challenges that require high energy levels (26-28). Although ERR $\alpha$ has been implicated in osteoclastogenesis (26, 27), a role for ERR $\alpha$ in mediating the effects of MYC on cell phenotype and metabolism has not been previously appreciated, and mechanisms by which ERR $\alpha$ promotes osteoclastogenesis are mostly unknown. We directly tested the role of MYC in inducing expression of Esrra, which encodes ERR $\alpha$. Induction of ERR $\alpha$ by RANKL (Figure 4, B and C, and Supplemental Figure 11, A and $\mathrm{B})$ was abrogated in both mouse and human MYC-deficient OCPs (Figure 4, B and C, and Supplemental Figure 11C). Of note, MYC-deficient human OCPs had impaired osteoclastogenesis (Supplemental Figure 11D). Compromised ERR $\alpha$ expression in MYC-deficient OCPs was restored by the reintroduction of MYC using retroviral transduction (Figure 4D), indicating that the expression of ERR $\alpha$ is dependent on MYC. In contrast, the expression of PGC1 $\beta$, a well-known ERR $\alpha$ coactivator, was not altered by the overexpression of MYC (Figure 4D), and MYC deficiency marginally decreased PGC1 $\beta$ expression (Figure $4 \mathrm{C}$ ). RANKL-induced expression of ERR $\alpha$ target genes was strongly attenuated in MYC-deficient OCPs (Supplemental Figure 12A). ChIP assays showed that RANKL induced the recruitment of MYC to the Esrra promoter (Supplemental Figure 12B) and that Esrra promoter activity was dependent on MYC (Supplemental Figure 12C). Taken together, these results show that MYC directly regulates ERR $\alpha$ in osteoclasts.

The MYC/ERR a axis regulates mitochondrial respiration in osteoclastogenesis. Consistent with previous reports using genetic approaches $(19,21), \mathrm{ERR} \alpha$ deficiency and treatment with XCT790 (29), an ERR $\alpha$ inhibitor, suppressed RANKL-induced osteoclast differentiation (Supplemental Figure 13 and Supplemental Figure 14A). Importantly, forced MYC expression, which restored osteoclast differentiation in MYC-deficient OCPs (Figure 2D and Figure 5A, left panels), was not able to restore osteoclast differentiation when ERR $\alpha$ was inhibited by XCT790 (Figure 5A). Taken together, these results identify a link between MYC and ERR $\alpha$ in osteoclasts and establish that ERR $\alpha$ is required for the effects of MYC on osteoclastogenesis.

ERR $\alpha$ transcriptionally regulates the expression of genes encoding energy metabolism regulators $(28,30,31)$. To test whether ERR $\alpha$ could functionally phenocopy the role of MYC in mitochondrial respiration in osteoclasts, we measured mitochondrial respiration following RANKL stimulation using ERR $\alpha$-deficient OCPs isolated from ERR $\alpha$-null mice. Indeed, ERR $\alpha$-deficient OCPs had a diminished RANKL-induced basal OCR and other mitochondrial activities (Figure 5, B and C). Consistently, expression of RANKL-inducible genes involving oxidative phosphorylation was significantly abrogated in ERR $\alpha$-deficient or XCT790-treated OCPs (Figure 5D). These results strongly support a role for a novel MYC/ERR $\alpha$ pathway in RANKL-activated mitochondrial respiration.

We next investigated whether overexpression of ERR $\alpha$ could restore impaired osteoclastogenesis in MYC-deficient OCPs. MYC-deficient OCPs were transduced with a retrovirus encoding ERR $\alpha$-turboGFP (ERR $\alpha$-tGFP), and the expression of ERR $\alpha$-tGFP was analyzed by immunoblotting (Supplemental Figure 15B). Ectopic expression of ERR $\alpha$ had a minimal effect on osteoclastogenesis in MYC-deficient OCPs (Supplemental Figure 15), which was similar to the inability of NFATc1 alone to rescue osteoclastogenesis in MYC-deficient OCPs (Figure 2F). Although ERR $\alpha$-deficient osteoclasts completely failed to resorb bone (Supplemental Figure 14B), RANKL-induced expression of NFATc1 and $\beta 3$ integrin in ERR $\alpha$-deficient osteoclasts was comparable to that in WT osteoclasts (Supplemental Figure 14C), supporting the notion that ERR $\alpha$ is not necessary for NFATc1 expression. Therefore, we tested whether both NFATc1 and ERR $\alpha$ are required for MYC-mediated osteoclastogenesis. Indeed, coexpression of Ca-NFATc1 and ERR $\alpha$ was able to partly rescue the impaired osteoclastogenesis in MYC-deficient OCPs (Figure 5E and Supplemental Figure 16). Collectively, our results suggest that ERR $\alpha$ and NFATc1 regulate distinct aspects of osteoclast differentiation and need to cooperate for effective osteoclastogenesis.

Targeting of the MYC/ERR $\alpha$ pathway protects mice against pathological bone loss. To test the importance of the MYC/ERR $\alpha$ pathway in pathological bone resorption and the efficacy of therapeutic targeting of this pathway to prevent bone loss for translational implications, we used the ovariectomy (OVX) model of estrogen

\section{Table 2. Motif analysis with GSEA using MYC-dependent genes on day 2 after RANIKL stimulation}

$\begin{array}{lcc}\text { Transcription factor } & \text { Binding motif } & \boldsymbol{P} \text { value } \\ \text { SP1 } & \text { GGGCGGR } & 3.71 \times 10^{-34} \\ \text { ERR } & \text { TCACCTY } & 7.9 \times 10^{-27} \\ \text { MAZ } & \text { GGGAGGRR } & 3.54 \times 10^{-19} \\ \text { SF1 } & \text { TCACCTTC } & 1.19 \times 10^{-18} \\ \text { NFAT } & \text { TGGAAA } & 1.28 \times 10^{-16} \\ \text { MYC } & \text { CACGTG } & 6.19 \times 10^{-14}\end{array}$

The 5 most enriched transcription factor-binding motifs in a \pm 2 -kb region of the transcriptional start site in MYC-dependent, RANKL-inducible genes $\left(P<10^{-14}\right)$. 
A

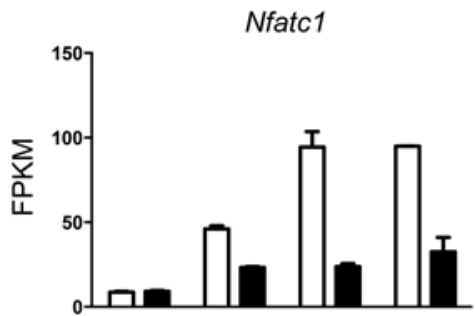

Sf1

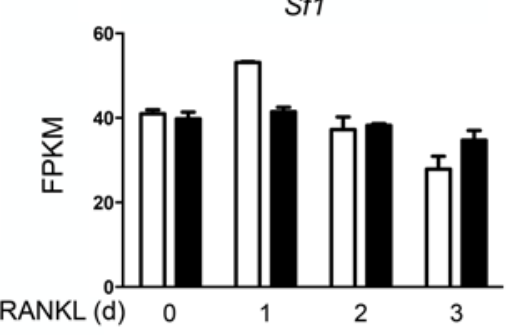

B

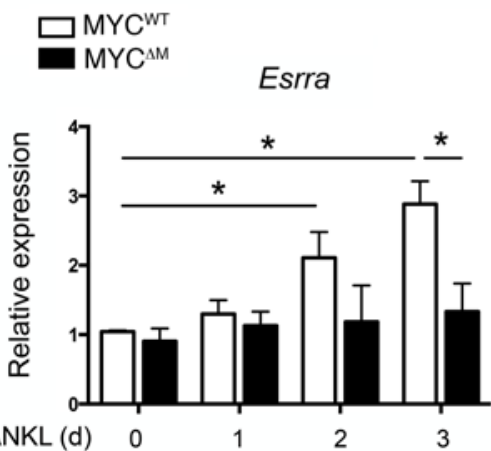

Esrra

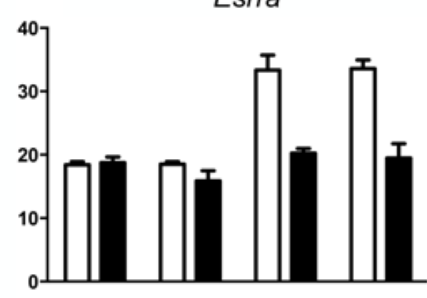

Maz

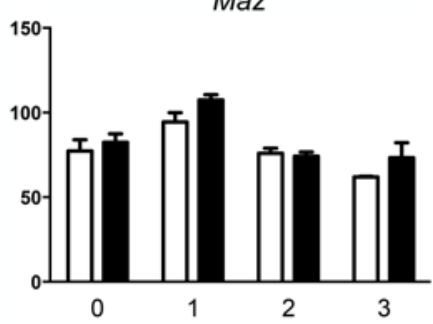

Myc

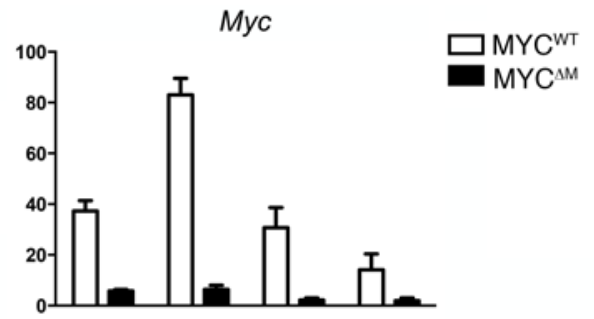

Sp1

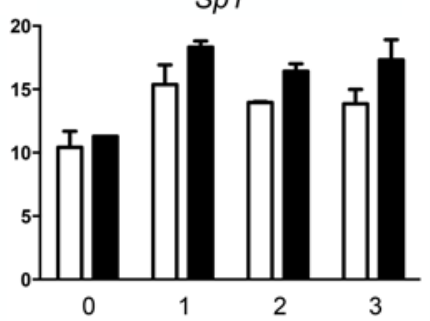

c

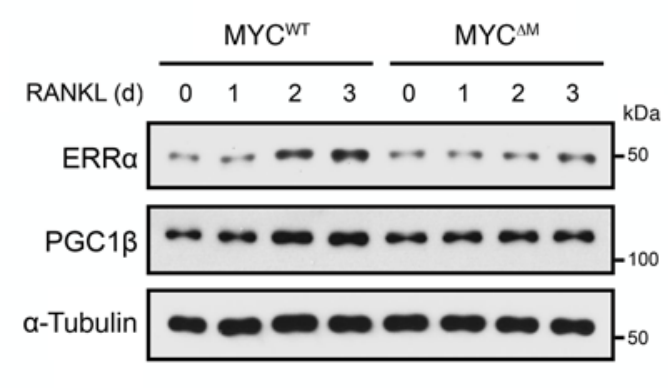

D

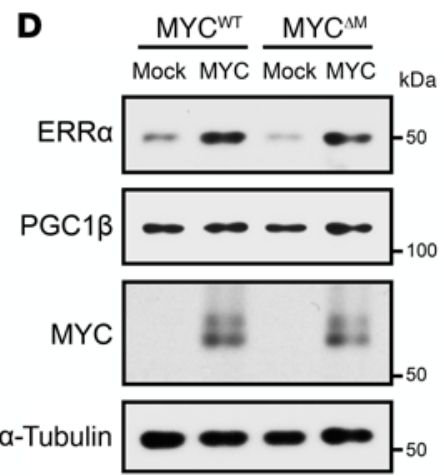

Figure 4. MYC regulates ERRa expression in osteoclastogenesis. (A) RNA-seq FPKM values for Nfatc1, Esrra, Myc, Sf1, Maz, and Sp1 in control (MYCWT) and MYC-deficient (MYC ${ }^{\triangle M}$ ) OCPs during osteoclast differentiation. RNA-seq data from 2 biological replicates are shown, and the level of each gene expression in each sample was normalized by means of FPKM. Data are shown as the mean \pm SEM. (B and C) Expression of ERR $\alpha$ in MYC ${ }^{W T}$ and MYC ${ }^{\triangle M}$ OCPs during osteoclastogenesis. (B) mRNA expression of Esrra (relative to the Hprt housekeeping gene), as measured by real-time quantitative PCR ( $n=6)$. Data are shown as the mean \pm SEM. ${ }^{*} P<0.05$, by 2-way ANOVA with Tukey's post-hoc test. (C) Protein expression of ERR $\alpha$ and PGC1 $\beta$, as determined by immunoblotting. Data are representative of 3 experiments. (D) Effect of MYC overexpression on ERR $\alpha$ and PGC1 $\beta$ expression levels. Protein expression of ERR $\alpha$ in mock-infected or MYC-transduced cells was determined by immunoblotting. Data are representative of 3 experiments.

deficiency-mediated bone loss (Figure 6A). Strikingly, $\mu \mathrm{CT}$ analysis revealed that $\mathrm{MYC}^{\Delta \mathrm{M}}$ mice were almost completely protected against OVX-induced bone loss, whereas $\mathrm{MYC}^{\mathrm{WT}}$ mice showed significant bone loss 6 weeks after OVX (Figure 6, B and C). Bone histomorphometric analysis also revealed a significant decrease in osteoclast numbers in $\mathrm{MYC}^{\Delta \mathrm{M}} \mathrm{OVX}$ mice compared with numbers in MYCWT OVX mice (Figure 6D). These results suggest that MYC in osteoclasts plays a crucial role in pathological bone resorption and that MYC is a potential therapeutic target for osteoporosis.

However, given the inherent structure of MYC, it is difficult to design a small-molecule inhibitor that directly binds to MYC $(32,33)$. Thus, we hypothesized that inhibiting downstream effector molecules such as ERR $\alpha$ would be an alternative method for suppressing MYC function. To test whether inhibition of ERR $\alpha$ shows a similar therapeutic efficacy in the treatment of pathological bone loss, we treated OVX mice with XCT790, a small-molecule inhibitor of ERR $\alpha$, in the OVX osteoporosis model using a therapeutic experimental design. XCT790 treatment was started
3 weeks after OVX and continued for 6 weeks (Figure 7A). To test the efficacy of XCT790 on osteoporosis, we used two different mouse strains with different basal bone density, one with high basal bone mass $(26.12 \% \pm 5.12 \%$; Figure $7, \mathrm{C}$ and $\mathrm{D})$ and the other with low basal bone mass $(13.30 \% \pm 2.66 \%$; Figure 7 , D and E), to account for strain-to-strain variations. XCT790 treatment significantly protected both strains of mice from OVX-induced bone loss (Figure 7, B-E), although the effect of XCT790 was milder than that seen with MYC deficiency. In addition, XCT790 treatment in sham-operated mice had a marginal effect on in vivo bone phenotype and formation, as measured by bone volume, bone formation rate (BFR), and mineral apposition rate (MAR) (Supplemental Figure 17, A-D). Consistently, we also found that XCT790 did not enhance in vitro osteoblastogenesis or activity at the dosage that suppressed osteoclastogenesis (Supplemental Figure 17, E-H). The expression of Runt-related transcription factor 2 (Runx2) was comparable between XCT790- and vehicle-treated cells (Supplemental Figure 17I). Taken together, these results suggest that 

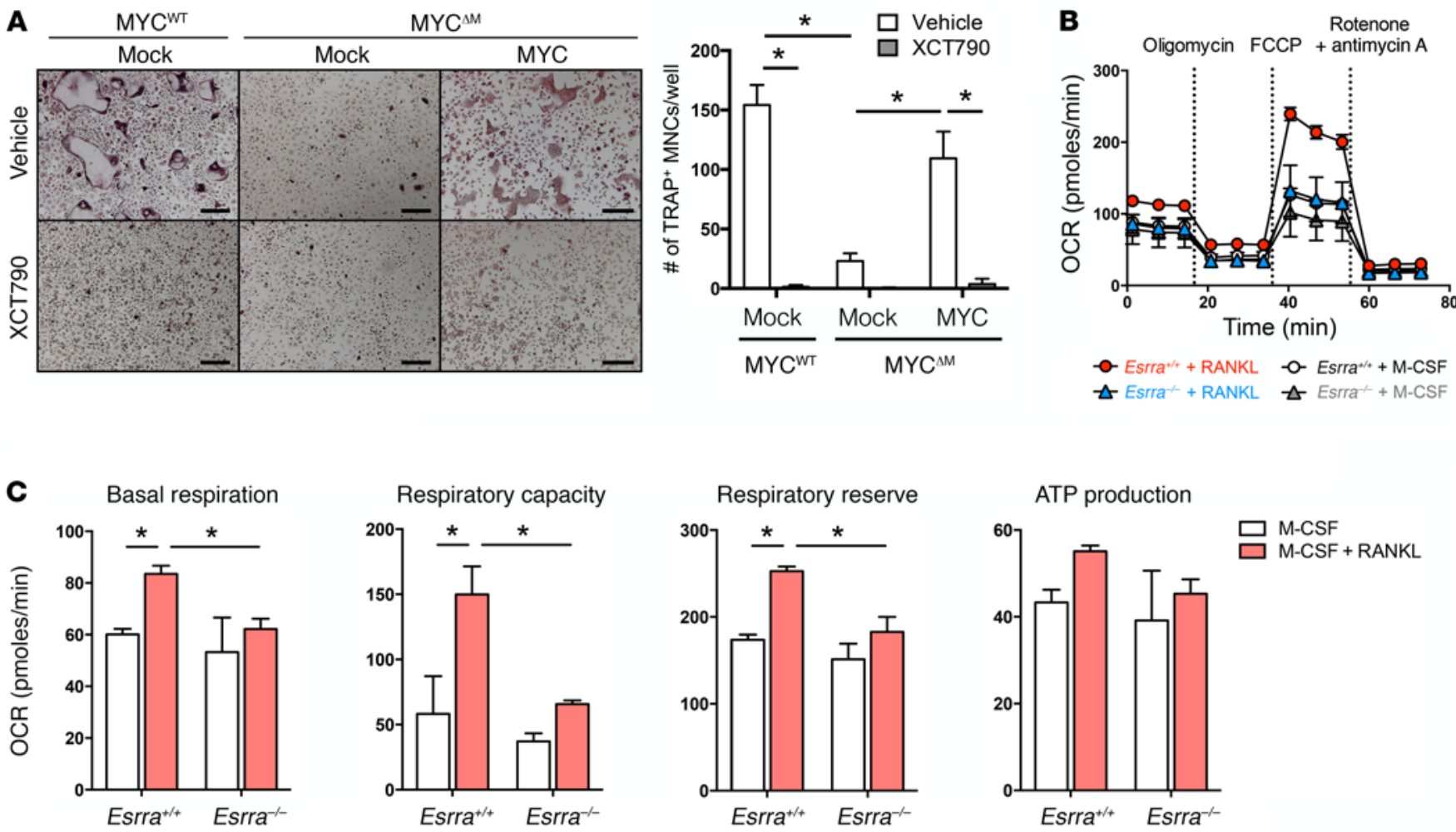

D

Idh3a

Sdhd

Cyc1

Cox $5 b$

Uqcrc2
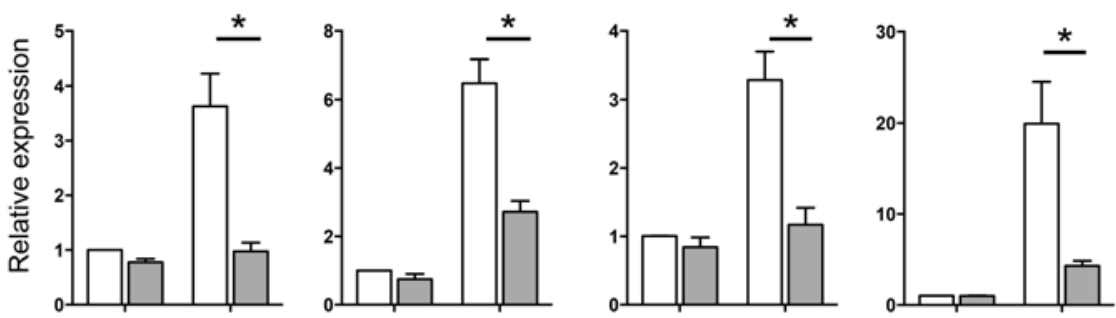

* $\square$ Esrra $^{* /+}$
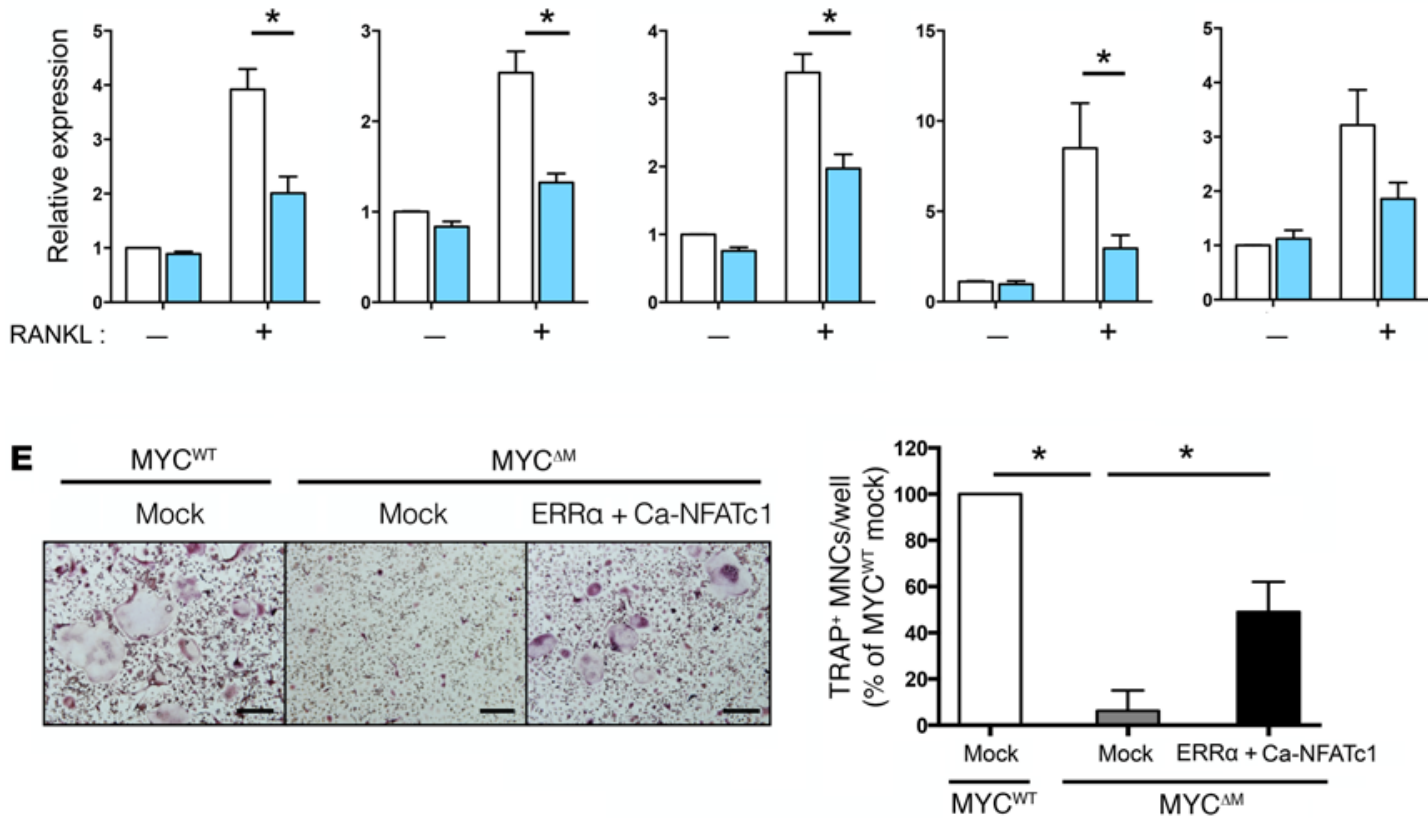
Figure 5. MYC-dependent expression of ERR $\alpha$ is required for osteoclastogenesis. (A) Osteoclast differentiation in mock-infected or MYC-transduced MYC ${ }^{\triangle M}$ OCPs treated with XCT790 $(10 \mu \mathrm{M})$. Representative images of TRAP-stained cells are shown. Scale bars: $200 \mu \mathrm{m}$. Graph indicates the number of TRAP-positive MNCs counted in triplicate from at least 3 experiments. (B and $\mathbf{C}$ ) Effect of ERR $\alpha$ deficiency on mitochondrial respiration. Control $\left(\mathrm{Esrra}^{+/+}\right)$and ERR $\alpha$-deficient $\left(\mathrm{Esrra}^{-/}\right)$OCPs were stimulated with RANKL ( $50 \mathrm{ng} / \mathrm{ml}$ ) for 2 days, and then the mitochondrial respiration rate of those cells was assessed by real-time measurement of the OCR ( $n=$ 4). (B) Representative time course data. (C) Assessment of mitochondrial activity as described in Supplemental Figure 9. (D) mRNA expression of oxidative phosphorylation-related genes in $\mathrm{Esrra}^{+/+}$and $\mathrm{Esrra}^{-/-}$OCPs and XCT790-treated OCPs $(10 \mu \mathrm{M})$ following RANKL stimulation ( $n=$ 3). Idh $3 a$, isocitrate dehydrogenase 3 ; Sdhd, succinate dehydrogenase complex, subunit D, integral membrane protein; Cyc1, cytochrome c-1; Cox5b, cytochrome c oxidase subunit Vb; Uacrc2, ubiquinol cytochrome c reductase core protein 2 . (E) Osteoclast differentiation in mock-infected or Ca-NFATC1- and ERR $\alpha$-transduced MYC ${ }^{\triangle M}$ OCPs. Representative images of TRAP-stained cells are shown. Scale bars: $200 \mu \mathrm{m}$. Graph shows the number of TRAP-positive MNCs counted in triplicate from 4 independent experiments. All data are shown as the mean \pm SEM. ${ }^{*} P<0.05$, by 2 -way ANOVA with Tukey's post-hoc test (A, C, and $\mathbf{D}$ ) and 1-way ANOVA with Tukey's post-hoc test (E).

inhibition of the MYC/ERR $\alpha$ pathway holds promise for the treatment of bone resorption, especially in conditions associated with increased MYC expression in OCPs.

\section{Discussion}

Constraining accelerated bone-resorbing osteoclast differentiation is an effective strategy for the treatment of osteoporosis (34-37). Our study highlights the importance of MYC in physiological and pathological bone remodeling and identifies a MYC/ $\mathrm{ERR} \alpha$ pathway as an important regulator of oxidative metabolism in osteoclasts. Moreover, targeting ERR $\alpha$ by XCT790 attenuated OVX-induced bone loss, an established model for human osteoporosis. These findings highlight the importance of the MYC/ERRa pathway as a potential therapeutic target with translational potential to treat pathological bone resorption.

It is generally accepted that MYC plays an essential role in metabolic mechanisms. Our study shows that MYC is a key determinant of metabolic reprogramming in osteoclasts. Recent studies focused on the role of MYC in metabolism reveal that MYC regulates activation-induced glycolysis and glutaminolysis in $\mathrm{T}$ cells (38), and inhibition of MYC-MAX interaction suppresses glutamine metabolism in in vitro osteoclastogenesis (7). However, how MYC integrates anabolic metabolism into different cellular functions in a cell type-specific manner is not clear. Our work demonstrates how alteration of metabolic pathways by MYC impacts physiological and pathological bone resorption by osteoclasts. Therefore, our results provide new insights into the role of MYC in metabolic reprogramming during osteoclastogenesis, which has been less studied.

Understanding the molecular determinants of metabolic reprogramming in osteoclasts is important, but the metabolic regulation in osteoclast differentiation is not completely elucidated. Recent studies have shown that effective osteoclastogenesis requires metabolic regulation including glycolytic flux and oxidative phosphorylation $(7,14)$. Our study adds another layer of complexity in metabolic reprogramming by demonstrat- ing the essential role of MYC in meeting energy and anabolic demands during osteoclast differentiation. In previous studies, increased oxidative phosphorylation has been partially mediated by increased mitochondrial biogenesis orchestrated by PGC1 $\beta$ (18) and is accompanied by an increase in S-adenosylmethionine (SAM) production (16). We have observed that the effect of MYC on oxidative phosphorylation is independent of mitochondrial biogenesis and that the overexpression of MYC is unable to regulate PGC1 $\beta$ expression, which suggests the uncoupling of PGC1 $\beta$ from oxidative phosphorylation in osteoclastogenesis. Supporting our observation, a recent study has also shown that the alternative NF- $\kappa \mathrm{B}$ pathway induced by RANKL regulates osteoclastogenesis independently of mitochondrial biogenesis (17) and that PGC1 $\beta$ deficient mice have a normal bone phenotype (19). Taken together, these results suggest that mitochondrial biogenesis is not a prerequisite for osteoclast formation and function.

Furthermore, our data establish MYC as a central regulator of oxidative metabolism in osteoclasts and place MYC at an upstream node in RANKL-induced osteoclastogenic signaling, where it is required for NFATc1 induction and the induction of respiratory metabolism mediated, in part, by ERR $\alpha$. Although the coexpression of NFATc1 and ERR $\alpha$ substantially rescued defective osteoclastogenesis in MYC-deficient OCPs, the incomplete restoration suggests that additional pathways downstream of MYC may play a role in osteoclastogenesis. Our GSEA pathway analysis further revealed that MYC is involved in other processes such as the TCA cycle, but its role in the TCA cycle in osteoclasts is poorly defined. Thus, several pathways regulated by MYC in osteoclasts are likely to exist, and further studies to fully reveal the role of MYC in osteoclasts will be necessary.

Our transcriptional data reveal that MYC regulates metabolic pathways through its downstream target, ERR $\alpha$, during osteoclastogenesis. ERR $\alpha$ has been shown to be important for osteoclastogenesis $(19,21)$, but the exact mechanism by which ERR $\alpha$ regulates osteoclast differentiation has remained unknown. We have shown, for the first time to our knowledge, a tight association between oxidative phosphorylation and the MYC/ERR $\alpha$ pathway during osteoclastogenesis. Emerging evidence has indicated that the induction of anabolic metabolic pathways by RANKL is important for osteoclastogenesis $(7,14-17)$, but the mechanisms by which these pathways are induced and their relationship to the NFATc1 program have not been clearly identified. We determined that ERR $\alpha$ deficiency results not only in defective oxidative phosphorylation but also in compromised bone resorption, without affecting RANKL-induced NFATc1 expression. In addition, either ERR $\alpha$ alone or NFATc1 alone did not rescue the impaired osteoclastogenesis seen in MYC-deficient OCPs. Although the importance of RANKL-induced NFATc1 as a master regulator of osteoclastogenesis is well established (11), our data suggest that the MYC/ERR $\alpha$ pathway described here is independent of, and works together with, NFATc1 to drive osteoclastogenesis.

Additionally, the deficiency of MYC in osteoclasts completely abrogates in vitro osteoclastogenesis and significantly protects mice against OVX-induced bone loss in vivo. Our results show that bone mass in $\mathrm{MYC}^{\Delta \mathrm{M}}$ mice was significantly increased (37\%) compared with that of control mice at 12 weeks. However, despite the substantial decrease in the number of osteoclasts, aging com- 
A

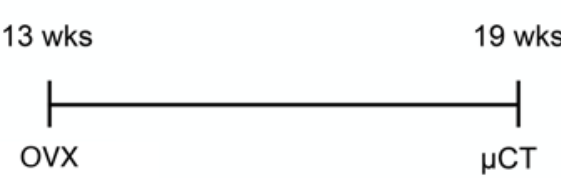

B

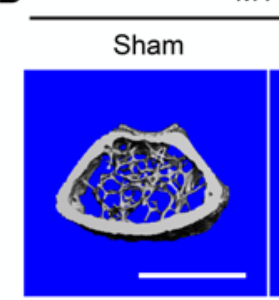

$\mathrm{MYC}^{\mathrm{WT}}$

OVX

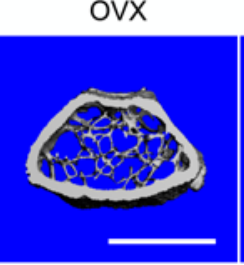

$\mathrm{MYC}^{\mathrm{MM}}$

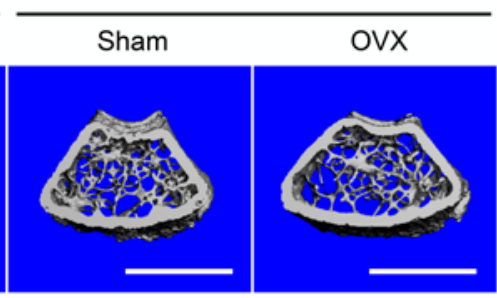

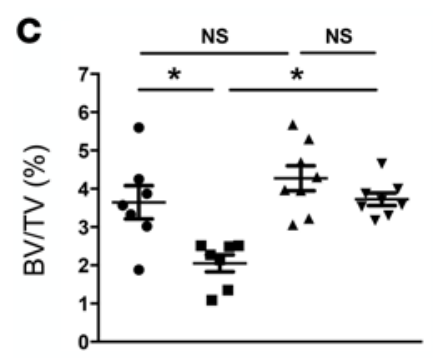

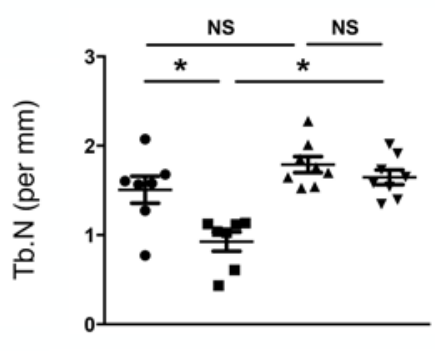

D
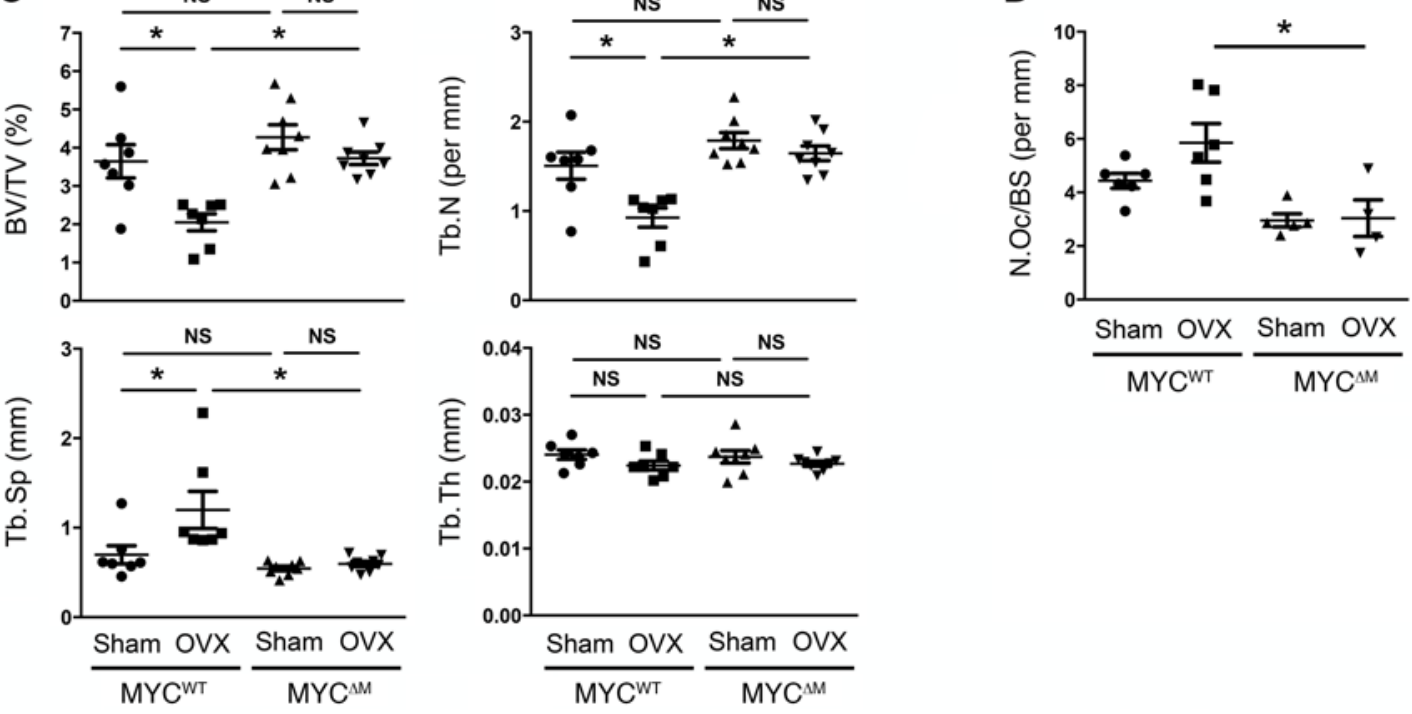

Figure 6. Ablation of MYC protects mice from OVX-induced pathological bone loss. (A) Schematic diagram illustrating the experimental design. (B and C) $\mu \mathrm{CT}$ analysis of femurs from 19-week-old sham-operated or OVX myeloid-specific MYC-deficient mice (MYC ${ }^{\Delta M}$ ) and control mice (MYCWT). $n=7$ sham-operated or OVX MYCWT; $n=8$ sham-operated or OVX MYC ${ }^{\Delta \mathrm{M}}$ mice. (B) Representative images show trabecular architecture by $\mu \mathrm{CT}$ reconstruction in distal femurs. Scale bars: $1 \mathrm{~mm}$. (C) $\mu \mathrm{CT}$ measurements for the indicated parameters in distal femurs. Bone volume, trabecular numbers, trabecular thickness, and trabecular space were determined by $\mu \mathrm{CT}$ analysis. (D) Osteoclast numbers per bone surface were measured by histomorphometric analysis ( $n \geq 4$ ). All data are shown as the mean \pm SEM. ${ }^{*} P<0.05$, by 1 -way ANOVA with Tukey's post-hoc test.

pensated for the difference in bone mass between the 2 groups. The discrepancy between in vivo and in vitro osteoclastogenesis has also been observed in many studies, including the studies in $\mathrm{NF}-\kappa \mathrm{B}-$ inducing kinase-deficient (NIK-deficient) and RelB proto-oncogene, NF- $\mathrm{KB}$ subunit-deficient (RelB-deficient) mice (17, 39). The absence of NIK or RelB in osteoclasts completely suppresses in vitro osteoclastogenesis and pathological osteolysis in the context of inflammation and bone metastasis, but has a minimal effect on basal bone phenotype $(40,41)$. Our study suggests that MYC may play a more prominent role in pathological conditions with accelerated bone loss such as osteoporosis than in physiological bone remodeling and that additional signals could compensate for impaired osteoclast activity in $\mathrm{MYC}^{\Delta \mathrm{M}}$ mice in vivo.

Our in vitro results from human blood-derived OCPs recapitulate our observations in murine OCPs, in that both showed the important role of MYC in osteoclasts, although the pattern of MYC expression or alteration of metabolic mechanisms in hyperactive osteoclasts during the pathogenesis of human osteoporosis is not known. Pathological conditions associated with excessive bone destruction include many diseases such as osteoporosis, rheumatoid arthritis, cancer metastases, and periprosthetic osteolysis. However, the known and druggable molecular targets in osteo- clasts are limited, impeding the development of antiresorptive drugs with effective pipelines. We propose that, although MYC is considered undruggable, directly targeting signaling components downstream of MYC can be a strategy to block MYC functions in osteoclasts. ERR $\alpha$ is one of the downstream candidates of MYC, and its inhibitor, XCT790, suppresses osteoclastogenesis and bone loss in the murine post-OVX model of human osteoporosis. While $\mathrm{ERR} \alpha$ is important for osteoclast differentiation and activity, ERR $\alpha$ deficiency has been shown to promote bone formation in vivo $(42,43)$. However, in our study, treatment with XCT790 showed a lack of enhancement of osteoblast function in vivo. The difference between our results and those from ERR $\alpha$-null mice may be explained by the pharmacokinetics and efficiency of XCT790 or by the differences in dosing. Targeting of ERR $\alpha$ by specific small-molecule inhibitors that have an optimal therapeutic window and can suppress excessive bone resorption and promote bone formation may have beneficial effects on pathological bone loss.

In summary, we have identified MYC as a key upstream regulator of RANKL-induced metabolic reprogramming and demonstrated the importance of the MYC/ERR $\alpha$ axis in osteoclasts as an integrator of metabolic reprogramming to promote osteoclast differentiation in physiological and pathological bone resorption, 
A

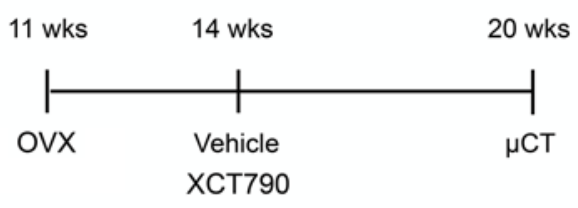

B High-bone-mass $\mathrm{C} 3 \mathrm{H}$ mice

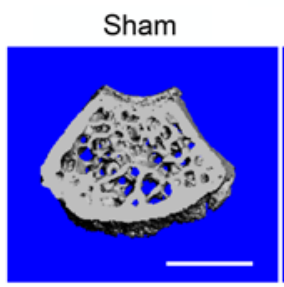

OVX

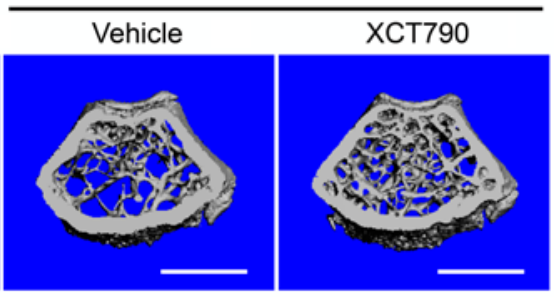

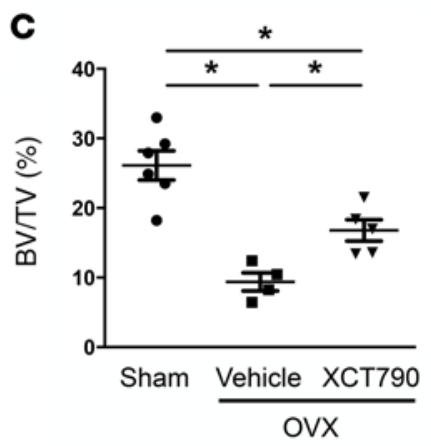
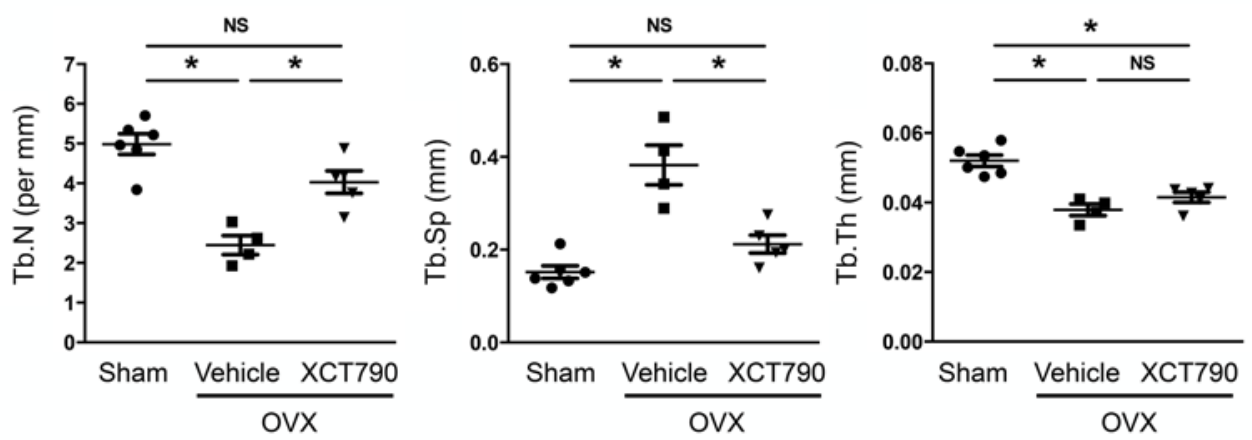

D Low-bone-mass CD-1 mice
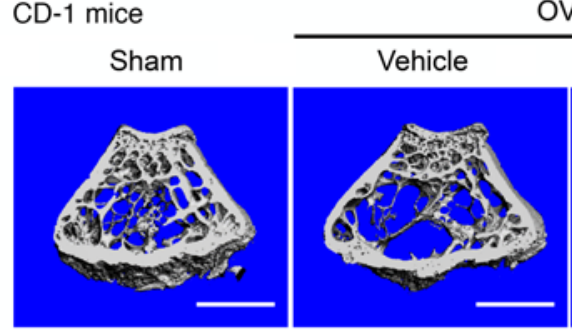

OVX
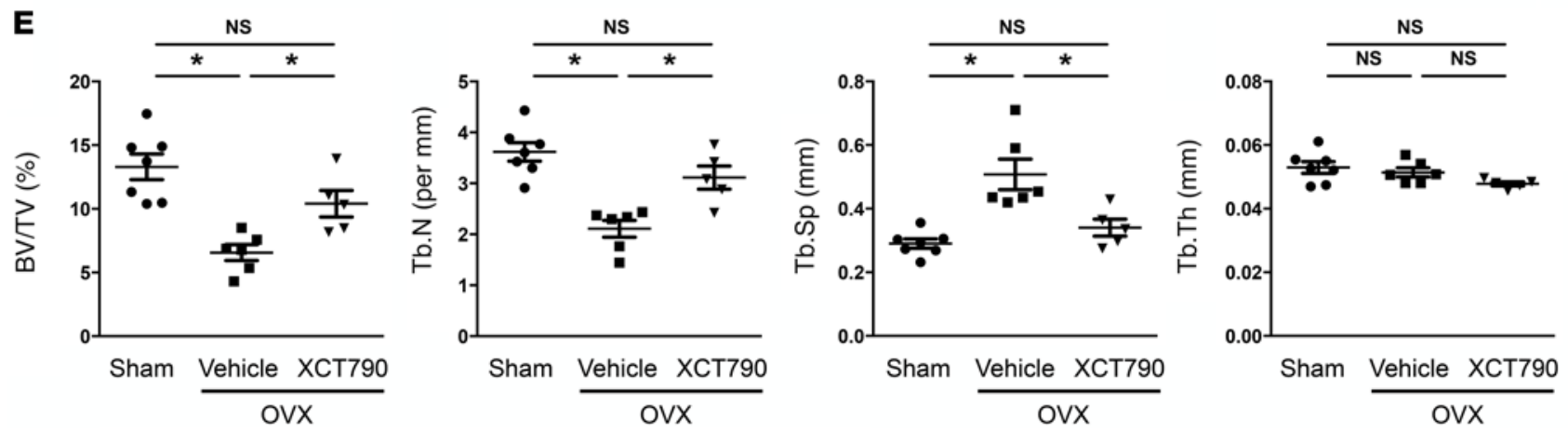

Figure 7. Therapeutic effect of XCT790 on the osteoporosis model. (A) Schematic diagram illustrating the experimental design. (B and C) $\mu C T$ analysis of the femurs of sham-operated, vehicle-treated (sham, $n=6$ ), vehicle-treated OVX (vehicle, $n=4$ ), and XCT790-treated OVX (XCT790, $n=5$ ) high-bonemass $\mathrm{C3H}$ mice. (B) Representative images showing trabecular architecture by $\mu \mathrm{CT}$ reconstruction in the distal femurs of C3H mice. Scale bars: 1 mm. (C) $\mu \mathrm{CT}$ measurements for the indicated parameters in distal femurs. ( $\mathbf{D}$ and $\mathbf{E}) \mu \mathrm{CT}$ analysis of the femurs of sham-operated, vehicle-treated (sham, $n=7$ ), vehicle-treated OVX (vehicle, $n=6$ ), and XCT790-treated OVX (XCT790, $n=5$ ) low-bone-mass CD-1 mice. (D) Representative images show trabecular architecture by $\mu \mathrm{CT}$ reconstruction in distal femurs. Scale bars: $1 \mathrm{~mm}$. (E) $\mu \mathrm{CT}$ measurements for the indicated parameters in distal femurs. All data are shown as the mean $\pm \mathrm{SEM}$. ${ }^{*} P<0.05$, by 1-way ANOVA with Tukey's post-hoc test.

opening up new lines of investigation to develop novel therapeutic interventions for pathological bone resorption.

\section{Methods}

Mice. All animals were maintained in a pathogen-free environment and were monitored by the investigators and staff at the Research
Animal Resource Center of the Weill Cornell Medical College. All animals were randomly assigned to experimental groups. We generated $\mathrm{MYC}^{\Delta \mathrm{M}}$ mice by crossing $M y c^{f / f l}$ mice (The Jackson Laboratory) with LysM-Cre (MYC ${ }^{\mathrm{WT}}$ ) mice (The Jackson Laboratory). All strains were on the C57BL/6/129 background. Littermate $\mathrm{MYC}^{\mathrm{WT}}$ mice were used as controls. For our MYC ${ }^{\Delta \mathrm{M}}$ mice bone phenotype study, 12-week-old 
male ( $n=11$ per group) and female ( $n=6$ per group) $\mathrm{MYC}^{\Delta \mathrm{M}}$ and littermate control $\mathrm{MYC}^{\mathrm{WT}}$ mice were used. For our OVX-induced bone loss study in $\mathrm{MYC}^{\Delta \mathrm{M}}$ mice, 13-week-old $\mathrm{MYC}^{\Delta \mathrm{M}}$ mice and their littermate $\mathrm{MYC}^{\mathrm{WT}}$ mice were randomly divided into sham-operated or ovariectomized groups ( $n \geq 7$ per group) and were housed in the facility for 6 weeks after the operation. To examine in vitro oxidative phosphorylation in the ERR $\alpha$-deficient condition, 8-week-old Esrra-deficient male mice and control mice were purchased from The Jackson Laboratory. To analyze the therapeutic effect of XCT790 (R\&D Systems), 11-week-old sham-operated or ovariectomized C3H mice $(n \geq 4$ per group) and CD- 1 mice ( $n \geq 5$ per group) from Charles River Laboratories were randomized and treated with either vehicle or XCT790 (5 $\mathrm{mg} / \mathrm{kg}$ for $\mathrm{C} 3 \mathrm{H}$ mice and $2.5 \mathrm{mg} / \mathrm{kg}$ for CD-1 mice) i.p. once per day for 6 weeks, beginning 3 weeks after the operation. XCT790 was prepared in $10 \%$ KLEPTOSE (Roquette Pharma) following the manufacturer's instructions.

Analysis of bone phenotype. $\mu \mathrm{CT}$ analysis (44) was performed as described previously (45), and all samples were included in the analysis, which was conducted in a blinded manner. For $\mu \mathrm{CT}$ analysis, a Scanco Medical $\mu$ CT 35 System with an isotropic voxel size of $7 \mu \mathrm{m}$ was used to image the distal femur. Scans were conducted in $70 \%$ ethanol and used an x-ray tube potential of $55 \mathrm{kVp}$, an x-ray intensity of $0.145 \mathrm{~mA}$, and an integration time of $600 \mathrm{~ms}$. For analysis of femoral bone mass, a 2.1-mm-wide region of trabecular bone was contoured, starting 280 $\mu \mathrm{m}$ from the proximal end of the distal femoral growth plate. A total of 250 slices were read in each sample. Femoral trabecular bone was thresholded at 211 per mille. Femoral cortical bone was thresholded at 350 per mille. A Gaussian noise filter optimized for murine bone was applied to reduce noise in the thresholded 2D image. 3D reconstructions were created by stacking the thresholded 2D images from the contoured region. For histopathological assessment, femurs were fixed in $4 \%$ paraformaldehyde for 3 days. These samples were decalcified with $10 \%$ neutral buffered EDTA (Sigma-Aldrich) and embedded in paraffin. To assess osteoclastogenesis and bone resorption, sections were stained with tartrate-resistant acid phosphatase (TRAP) and hematoxylin for osteoclast visualization on trabecular bone within the femur metaphysis. All measurements were performed using Osteometric software (Osteomeasure) according to standard procedures (46) and were confined to the secondary spongiosa and restricted to an area between 400 and 2,000 $\mathrm{mm}$ distal to the growth plate metaphyseal junction of the distal femur. Osteoclasts were identified as TRAP-positive cells that were multinucleated and adjacent to bone.

Reagents. Human M-CSF and RANKL were purchased from Peprotech. The ERR $\alpha$ inverse agonist XCT790 was purchased from R\&D Systems. The metabolic inhibitors 2-deoxy-D-glucose (2-DG), 6-diazo-5-oxo-L-norleucine (DON), and oligomycin, and the MYC inhibitor 10058-F4 were purchased from Sigma-Aldrich. The antibodies used for immunoblotting were as follows: NFATc1 (BD Pharmingen; 556602); MYC, ERR $\alpha$, and $\beta 3$ integrin (Cell Signaling Technology; 5605, 13826, and 4702); p38 (Santa Cruz Biotechnology Inc.; sc535); $\alpha$-tubulin (Sigma-Aldrich; T9026); lamin B and PGC1 $\beta$ (Abcam; ab16048 and ab176328); phosphorylated ERK1/2 (p-ERK1/2), IкB $\alpha$, and p-p38 (Cell Signaling Technology; 9101, 9242, and 9215); and tGFP (Origene; TA150041).

Osteoclast differentiation. Bone marrow cells were flushed from the femurs of mice, followed by lysis of rbc using ammonium chloride potassium (ACK) lysis buffer (Cambrex). The surviving cells were cul- tured in $\alpha$-MEM supplemented with 10\% FBS and 5\% L929 cell supernatant, which served as as a source of M-CSF (47). The nonadherent cell population was recovered the next day and cultured with M-CSFcontaining conditioned medium (CM) for 3 additional days. The final concentration of M-CSF in our culture was $23.3 \mathrm{ng} / \mathrm{ml}$. We defined this cell population as mouse OCPs. For osteoclastogenesis assays, mouse OCPs were plated at a seeding density of $1 \times 10^{4}$ per well in triplicate wells on a 96-well plate and incubated with M-CSF-containing CM and RANKL (50 ng/ml) for an additional 3 days, with exchange of fresh M-CSF-containing CM and RANKL every 2 days. Cells were fixed and stained for TRAP using the Acid Phosphatase Leukocyte Diagnostic Kit (Sigma-Aldrich) as recommended by the manufacturer. Multinucleated ( $>3$ nuclei) TRAP-positive osteoclasts were counted in triplicate wells.

Bone resorption pit assays. The bone resorption activity of osteoclasts was examined using 96-well Corning Osteo Assay Surface Plates (Sigma-Aldrich). Mouse OCPs were plated at a seeding density of $1 \times 10^{4}$ per well and incubated with M-CSF-containing CM and RANKL (50 ng/ml) for 10 days, with exchange of fresh M-CSF-containing CM and RANKL every 2 days. After removing cells with $10 \%$ bleach solution, plates were stained with $1 \%$ toluidine blue solution to visualize the formation of pits. The resorbed area was analyzed using OsteoMeasure software (OsteoMetrics).

Retroviral overexpression. The appropriate retroviral constructs as described in the Supplemental Methods were transfected into a packaging cell line, Plat-E, using FuGENE HD Transfection Reagent (Promega), and then the viral supernatant was collected after 48 hours of incubation. The filtered virus-containing supernatant was mixed with equal volumes of $\alpha$-MEM containing 20\% FBS in the presence of $6 \mu \mathrm{g} / \mathrm{ml}$ polybrene (Santa Cruz Biotechnology Inc.) along with $10 \%$ $\mathrm{M}-\mathrm{CSF}$-containing CM and then added to OCPs. After 24 hours of viral incubation, OCPs were used for experiments.

RNA preparation and real-time PCR. DNA-free RNA was obtained using the RNeasy Mini Kit (QIAGEN) with DNase treatment, and 0.5 $\mu \mathrm{g}$ total RNA was reverse transcribed using a First Strand cDNA Synthesis Kit (Thermo Fisher Scientific). Real-time PCR was performed in triplicate using the iCycler iQ Thermal Cycler and Detection System (Applied Biosystems) following the manufacturer's protocol. Expression of the tested gene was normalized relative to levels of hypoxanthine guanine phosphoribosyl transferase (Hprt) for mice and TATA box-binding protein $(T B P)$ for humans. The primer sequences are listed in Supplemental Table 1.

RNA-seq. Total RNA was first extracted. TruSeq Sample Preparation Kits (Illumina) were then used to purify poly-A transcripts and generate libraries with multiplexed barcode adaptors. All samples passed quality control analysis on a Bioanalyzer 2100 (Agilent Technologies). Paired-end reads $\left(50 \times 2\right.$ cycles, $\sim 75 \times 10^{6}$ reads per sample) were obtained on an Illumina HiSeq 2500 at the Weill Cornell Medical College Genomics Resources Core Facility. The TopHat program (version 2.0.11) was used to align the reads to the mm10 mouse reference genome, while the Cufflinks program (version 2.2.0) allowed for measurements of transcript abundance (represented by fragments per kilobase of exon model per million mapped reads [FPKM]). Genes and transcripts with FPKM values below 5 were not included in the analysis. Pearson's correlation analysis was performed, and heatmaps were generated using R (version 3.0.2). Data from 2 biological replicates were used for bioinformatics analysis. 
Mitochondrial oxygen consumption measurement. The real-time mitochondrial OCR and extracellular acidification rate (ECAR) were measured using the XF96 Extracellular Flux Analyzer (Seahorse Bioscience) with the Cell Mito Stress Kit (Seahorse Bioscience) following the manufacturer's instructions. The measurement was normalized to the relative level of DNA determined by measuring the fluorescence intensity of cells stained by SYTO 24 Green Fluorescent Nucleic Acid Stain (Molecular Probes). Briefly, OCPs were seeded on XF96 cell culture microplates (Seahorse Bioscience) at a seeding density of $8 \times 10^{3}$ cells per well and stimulated with RANKL ( $50 \mathrm{ng} / \mathrm{ml}$ ) for 2 days. Before the assay, cells were rinsed twice and kept in prewarmed XF assay medium ( $\mathrm{pH}$ 7.4) supplemented with $1 \mathrm{mM}$ sodium pyruvate, $2 \mathrm{mM}$ glutamine, and $10 \mathrm{mM}$ glucose in a $37^{\circ} \mathrm{C}$ non- $\mathrm{CO}_{2}$ incubator for 1 hour. Next, the respiratory rate was measured at $37^{\circ} \mathrm{C}$ in at least 3 replicates per condition by using the following perturbation drugs in succession: $1 \mu \mathrm{M}$ oligomycin, $2 \mu \mathrm{M}$ carbonyl cyanide- $p$ trifluoromethoxyphenylhydrazone (FCCP), and $0.5 \mu \mathrm{M}$ rotenone/antimycin A. The basal OCR was measured before drug exposure. We calculated the mitochondrial function metrics as described in Supplemental Figure 9 and as directed in the Cell Mito Stress Kit manual (Seahorse Bioscience).

Accession numbers. The RNA-seq data were deposited in the NCBI's Sequence Read Archive (SRA) under accession number SRP096890.

Statistics. All statistical analyses were performed with GraphPad Prism 5.0 (GraphPad Software) using a 2-tailed, unpaired $t$ test (2 conditions) or 1- or 2-way ANOVA for multiple comparisons (>2 conditions) with Tukey's post-hoc test. A $P$ value of less than 0.05 was considered statistically significant.
Study approval. All animal experiments were reviewed and approved by the IACUC of Weill Cornell Medical College.

\section{Author contributions}

SB and MJL conceived, designed, and performed most of the experiments, analyzed data, and wrote the manuscript. EGG contributed to and performed bioinformatics data analysis. VYG and JRC contributed to extracellular flux studies. SHM, KM, and MM contributed to bone phenotype analysis. VG contributed to ERR $\alpha$ experiments. KHPM conceived, designed, and oversaw the project and wrote the manuscript.

\section{Acknowledgments}

We thank Lionel B. Ivashkiv, Steven Goldring (Hospital for Special Surgery, New York, NY), Matthew B. Greenblatt (Weill Cornell Medical College, New York, NY) for helpful discussions and critical review of the manuscript and Jae-Hyuck Shim and Yeon Suk Yang (University of Massachusetts Medical School) for micro-CT analysis and helpful discussions. This study was supported by the National Institute of Arthritis and Musculoskeletal and Skin Diseases (NIAMS), NIH (R00AR061430 and R01AR069562). The contents of this manuscript are solely the responsibility of the authors and do not necessarily represent the official views of the NIH.

Address correspondence to: Kyung-Hyun Park-Min; 535 East 70th Street, New York, New York 10021, USA. Phone: 212.774.7631; Email:ParkminK@hss.edu.
1. Nie Z, et al. c-Myc is a universal amplifier of expressed genes in lymphocytes and embryonic stem cells. Cell. 2012;151(1):68-79.

2. Lin CY, et al. Transcriptional amplification in tumor cells with elevated c-Myc. Cell. 2012;151(1):56-67.

3. Rahl PB, et al. c-Myc regulates transcriptional pause release. Cell. 2010;141(3):432-445.

4. Baudino TA, et al. c-Myc is essential for vasculogenesis and angiogenesis during development and tumor progression. Genes Dev. 2002;16(19):2530-2543.

5. Dang CV. MYC on the path to cancer. Cell. 2012;149(1):22-35.

6. Battaglino R, Kim D, Fu J, Vaage B, Fu XY, Stashenko P. c-myc is required for osteoclast differentiation. J Bone Miner Res. 2002;17(5):763-773.

7. Indo Y, et al. Metabolic regulation of osteoclast differentiation and function. J Bone Miner Res. 2013;28(11):2392-2399.

8. Park-Min KH, et al. Inhibition of osteoclastogenesis and inflammatory bone resorption by targeting BET proteins and epigenetic regulation. Nat Commun. 2014;5:5418.

9. Takayanagi H. Osteoimmunology: shared mechanisms and crosstalk between the immune and bone systems. Nat Rev Immunol. 2007;7(4):292-304.

10. Lorenzo J, Horowitz M, Choi Y. Osteoimmunology: interactions of the bone and immune system. Endocr Rev. 2008;29(4):403-440.

11. Takayanagi $\mathrm{H}$. The role of NFAT in osteoclast formation. Ann N Y Acad Sci. 2007;1116:227-237.

12. Aliprantis AO, et al. NFATc1 in mice represses osteoprotegerin during osteoclastogenesis and dissociates systemic osteopenia from inflammation in cherubism. JClin Invest. 2008;118(11):3775-3789.

13. Charles JF, et al. The collection of NFATc1dependent transcripts in the osteoclast includes numerous genes non-essential to physiologic bone resorption. Bone. 2012;51(5):902-912.

14. Ikeda K, Takeshita S. The role of osteoclast differentiation and function in skeletal homeostasis. JBiochem. 2016;159(1):1-8.

15. Jin Z, Wei W, Yang M, Du Y, Wan Y. Mitochondrial complex I activity suppresses inflammation and enhances bone resorption by shifting macrophage-osteoclast polarization. Cell Metab. 2014;20(3):483-498.

16. Nishikawa K, et al. DNA methyltransferase 3a regulates osteoclast differentiation by coupling to an S-adenosylmethionine-producing metabolic pathway. Nat Med. 2015;21(3):281-287.

17. Zeng R, Faccio R, Novack DV. Alternative NF- $\mathrm{B}$ Regulates RANKL-Induced Osteoclast Differentiation and Mitochondrial Biogenesis via Independent Mechanisms. J Bone Miner Res. 2015;30(12):2287-2299.

18. Ishii KA, et al. Coordination of PGC-1beta and iron uptake in mitochondrial biogenesis and osteoclast activation. Nat Med. 2009;15(3):259-266.

19. Wei W, et al. PGC1beta mediates PPARgamma activation of osteoclastogenesis and rosiglitazone-induced bone loss. Cell Metab. 2010;11(6):503-516.

20. Wan Y. PPAR $\gamma$ in bone homeostasis. Trends Endocrinol Metab. 2010;21(12):722-728.

21. Wei W, et al. Ligand Activation of ERR $\alpha$ by Cho- lesterol Mediates Statin and Bisphosphonate Effects. Cell Metab. 2016;23(3):479-491.

22. Clausen BE, Burkhardt C, Reith W, Renkawitz $\mathrm{R}$, Förster I. Conditional gene targeting in macrophages and granulocytes using LysMcre mice. Transgenic Res. 1999;8(4):265-277.

23. Pello OM, et al. In vivo inhibition of c-MYC in myeloid cells impairs tumor-associated macrophage maturation and pro-tumoral activities. PLoS One. 2012;7(9):e45399.

24. Charles JF, Hsu LY, Niemi EC, Weiss A, Aliprantis AO, Nakamura MC. Inflammatory arthritis increases mouse osteoclast precursors with myeloid suppressor function. J Clin Invest. 2012;122(12):4592-4605

25. Subramanian A, et al. Gene set enrichment analysis: a knowledge-based approach for interpreting genome-wide expression profiles. Proc Natl Acad Sci USA. 2005;102(43):15545-15550.

26. Carnesecchi J, Vanacker JM. Estrogen-Related Receptors and the control of bone cell fate. $\mathrm{Mol}$ Cell Endocrinol. 2016;432:37-43.

27. Bonnelye E, Aubin JE. An energetic orphan in an endocrine tissue: a revised perspective of the function of estrogen receptor-related receptor alpha in bone and cartilage. J Bone Miner Res. 2013;28(2):225-233.

28. Huss JM, Garbacz WG, Xie W. Constitutive activities of estrogen-related receptors: Transcriptional regulation of metabolism by the ERR pathways in health and disease. Biochim Biophys Acta. 2015;1852(9):1912-1927.

29. Busch BB, et al. Identification of a selective inverse agonist for the orphan nuclear receptor 
estrogen-related receptor alpha. J Med Chem. 2004;47(23):5593-5596.

30. Villena JA, Kralli A. ERRalpha: a metabolic function for the oldest orphan. Trends Endocrinol Metab. 2008;19(8):269-276.

31. Yuk JM, et al. Orphan Nuclear Receptor ERR $\alpha$ Controls Macrophage Metabolic Signaling and A20 Expression to Negatively Regulate TLR-Induced Inflammation. Immunity. 2015;43(1):80-91.

32. Dang CV. Therapeutic targeting of Myc-reprogrammed cancer cell metabolism. Cold Spring Harb Symp Quant Biol. 2011;76:369-374.

33. Chen BJ, Wu YL, Tanaka Y, Zhang W. Small molecules targeting c-Myc oncogene: promising anti-cancer therapeutics. Int J Biol Sci. 2014;10(10):1084-1096.

34. Zaidi M. Skeletal remodeling in health and disease. Nat Med. 2007;13(7):791-801.

35. Raisz LG. Pathogenesis of osteoporosis: concepts, conflicts, and prospects. J Clin Invest. 2005;115(12):3318-3325.
36. Novack DV, Teitelbaum SL. The osteoclast: friend or foe? Annu Rev Pathol. 2008;3:457-484.

37. Rosen CJ. Clinical practice. Postmenopausal osteoporosis. N Engl JMed. 2005;353(6):595-603.

38. Wang R, et al. The transcription factor Myc controls metabolic reprogramming upon $\mathrm{T}$ lymphocyte activation. Immunity. 2011;35(6):871-882.

39. Novack DV, et al. The IkappaB function of NF-kappaB2 p100 controls stimulated osteoclastogenesis. JExp Med. 2003;198(5):771-781.

40. Vaira S, et al. RelB is the NF-kappaB subunit downstream of NIK responsible for osteoclast differentiation. Proc Natl Acad Sci U S A. 2008;105(10):3897-3902.

41. Yang C, et al. NIK stabilization in osteoclasts results in osteoporosis and enhanced inflammatory osteolysis. PLoS One. 2010;5(11):e15383.

42. Teyssier $\mathrm{C}$, et al. Absence of ERRalpha in female mice confers resistance to bone loss induced by age or estrogen-deficiency. PLoS One. 2009;4(11):e7942.
43. Gallet M, et al. Repression of osteoblast maturation by ERR $\alpha$ accounts for bone loss induced by estrogen deficiency. PLoS One. 2013;8(1):e54837.

44. Bouxsein ML, Boyd SK, Christiansen BA, Guldberg RE, Jepsen KJ, Müller R. Guidelines for assessment of bone microstructure in rodents using micro-computed tomography. J Bone Miner Res. 2010;25(7):1468-1486.

45. Shim JH, et al. Schnurri-3 regulates ERK downstream of WNT signaling in osteoblasts. JClin Invest. 2013;123(9):4010-4022.

46. Parfitt AM, et al. Bone histomorphometry: standardization of nomenclature, symbols, and units. Report of the ASBMR Histomorphometry Nomenclature Committee. J Bone Miner Res. 1987;2(6):595-610.

47. Englen MD, Valdez YE, Lehnert NM, Lehnert BE. Granulocyte/macrophage colony-stimulating factor is expressed and secreted in cultures of murine L929 cells. JImmunol Methods. 1995;184(2):281-283. 